\title{
Store-Operated Calcium Entry through Orai Is Required for Transcriptional Maturation of the Flight Circuit in Drosophila
}

\author{
Trayambak Pathak, ${ }^{1,2}$ Tarjani Agrawal, ${ }^{1}$ Shlesha Richhariya, ${ }^{1}{ }^{\circledR}$ Sufia Sadaf, ${ }^{1}$ and $\bigodot_{\text {Gaiti Hasan }}{ }^{1}$ \\ ${ }^{1}$ National Centre for Biological Sciences, Tata Institute of Fundamental Research, Bangalore 560065, India, and ${ }^{2}$ Manipal University, Manipal, Karnataka \\ 576104, India
}

\begin{abstract}
Store operated calcium entry (SOCE) is thought to primarily regulate calcium homeostasis in neurons. Subsequent to identification of Orai as the SOCE channel in nonexcitable cells, investigation of Orai function in neurons demonstrated a requirement for SOCE in Drosophila flight. Here, by analysis of an Orai mutant and by controlled expression of a dominant-negative Drosophila Orai transgene, we show that Orai-mediated SOCE is required in dopaminergic interneurons of the flight circuit during pupal development. Expression of dominant-negative Orai in dopaminergic neurons of pupae abolished flight. The loss of Orai-mediated SOCE alters transcriptional regulation of dopaminergic neurons, leading to downregulation of the enzyme tyrosine hydroxylase, which is essential for dopamine synthesis, and the dopamine transporter, which is required for dopamine uptake after synaptic release. These studies suggest that modulation of SOCE could serve as a novel mechanism for restoring dopamine levels in dopaminergic neurons.
\end{abstract}

Key words: dopamine transporter; dopaminergic neurons; IP3R; STIM; tyrosine hydroxylase

\section{Significance Statement}

The specificity of an animal's response to an environmental stimulus is determined in part by the release of neurotransmitters, which are sensed by responding neurons through cognate receptors on their surface. One way by which neurons respond is through release of calcium from intracellular stores followed by store refilling from extracellular calcium sources. This mechanism is called store-operated calcium entry (SOCE). The function of SOCE in neurons has been debated. Here we describe a new function for SOCE in the regulation of neurotransmitter levels in Drosophila flight neurons. This cell-signaling mechanism is required to maintain optimal levels of a key enzyme for dopamine synthesis and may serve as a mechanism for restoring dopamine levels in relevant pathological conditions.

\section{Introduction}

Neural circuits arrive at their pattern of adult connections through genetically encoded developmental programs, which define the identity of individual neurons, and neuronal activity during circuit maturation (Berridge, 1998; Spitzer, 2002; Borodinsky

\footnotetext{
Received April 30, 2015; revised Aug. 28, 2015; accepted Aug. 30, 2015.

Author contributions: T.P. and G.H. designed research; T.P., T.A., S.R., and S.S. performed research; T.P. and G.H. contributed unpublished reagents/analytic tools; T.P., T.A., S.R., and S.S. analyzed data; T.P. and G.H. wrote the paper.

This project was supported by grants from India's Department of Science \& Technology (http://www.dst.gov.in) and National Centre for Biological Sciences (NCBS), Tata Institute of Fundamental Research (http://www.ncbs.res. in) to G.H., T.P. is supported by a research fellowship from India's Council of Scientific and Industrial Research (http://www.csir.res.in). Stocks obtained from the Bloomington Drosophila Stock Center (National Institutes of Health P400D018537) were used in this study. Bacterial expression of dOrai, for antibody generation, was obtained with the help of Dr. Deepak Nair's laboratory at NCBS. We thank Dr. Krishnamurthy and NCBS Central Imaging and Flow Facility for help with confocal imaging and FACS of primary neurons, and the Fly Facility, Centre for Cellular and Molecular Platforms, NCBS for generating transgenic fly lines.
}

et al., 2004). In vertebrates, neuronal activity followed by entry of extracellular $\mathrm{Ca}^{2+}$ has been shown to influence multiple aspects of neural function by activating target genes that play important roles in cell survival, dendritic and axonal growth, synaptic development, and neuronal plasticity (Berridge, 1998). In addition to activity-dependent mechanisms of $\mathrm{Ca}^{2+}$ entry channels, vertebrate neurons also express STIM 1 and 2 (Skibinska-Kijek et al., 2009) and Orai (Somasundaram et al., 2014), the recently identified components of store-operated $\mathrm{Ca}^{2+}$ entry (SOCE; Feske et al., 2006; Vig et al., 2006). Knockdown of STIM and Orai in cultured neural progenitor cells suggests that SOCE-dependent transcriptional changes could regulate mammalian neurogenesis

\footnotetext{
The authors declare no competing financial interests.

Correspondence should be addressed to Gaiti Hasan at the above address. E-mail: gaiti@ncbs.res.in.

DOl:10.1523/JNEUROSCI.1680-15.2015

Copyright $\odot 2015$ the authors $\quad 0270-6474 / 15 / 3513784-16 \$ 15.00 / 0$
} 
(Somasundaram et al., 2014). In the mature brain, SOCE is thought to be required for the maintenance of neuronal $\mathrm{Ca}^{2+}$ homeostasis, which in turn can influence synaptic transmission and plasticity (Hartmann et al., 2014). Whereas some of these changes arise from a direct effect on endoplasmic reticular (ER) store $\mathrm{Ca}^{2+}$ (Hartmann et al., 2014), other changes might arise through regulation of transcription in resting neurons (Lalonde et al., 2014) and thus possible modulation of presynaptic and postsynaptic function. Neuronal activity also affects dendritic and synaptic growth and function in Drosophila (Miller et al., 2012; Timmerman et al., 2013; Vonhoff et al., 2013; Ryglewski et al., 2014). To identify molecular mechanisms that underlie the changes brought about by reduced SOCE in neural development, we investigated Drosophila Orai (dOrai) mutants for behavioral, cellular, and molecular deficits.

Previous work has shown that loss of inositol 1,4,5-trisphosphate receptor $\left(\mathrm{IP}_{3} \mathrm{R}\right)$ function and knockdown of either $\mathrm{dSTIM}$ or dOrai in Drosophila neurons specifically affects the ability to initiate and maintain flight (Banerjee et al., 2004; Venkiteswaran and Hasan, 2009; Agrawal et al., 2010). Moreover, flight deficits in $\mathrm{IP}_{3} \mathrm{R}$ mutant flies could be rescued by hypermorphic alleles of dOrai and by overexpression of dSTIM in neurons (Venkiteswaran and Hasan, 2009). These observations support the idea that in Drosophila neurons, $\mathrm{Ca}^{2+}$ release from ER stores through the $\mathrm{IP}_{3} \mathrm{R}$ triggers SOCE through STIM and Orai (Venkiteswaran and Hasan, 2009). A similar signaling mechanism exists in mammalian Purkinje neurons (Wu et al., 2011; Hartmann et al., 2014). Here we investigated the spatiotemporal requirement of SOCE for flight in Drosophila neurons and have identified a requirement for SOCE in dopaminergic neurons during pupal development. To investigate mechanism(s) by which SOCE affects flight, we tested an existing loss-of-function mutant for dOrai (Cuttell et al., 2008), which enabled us to confirm our previous findings with pan-neuronal knockdown of dOrai (Venkiteswaran and Hasan, 2009). To identify neurons that require SOCE for flight, we developed an inducible dominant-negative dOrai transgene (Yeromin et al., 2006). Expression of the dominantnegative dOrai transgene, $\mathrm{dOrai}^{\mathrm{E180A}}$ (E180A), specifically in dopaminergic neurons during pupal stages, abolished flight. Molecular analysis of E180A-expressing dopaminergic neurons indicates that SOCE helps establish and maintain their specific transcriptional profile during flight-circuit maturation.

\section{Materials and Methods}

Fly rearing and stocks. Drosophila strains were grown in corn flour agar media, which was supplemented with yeast. All strains were grown at $25^{\circ} \mathrm{C}$, unless otherwise specified in the text. The Drosophila strain Canton $S$ was used as the wild-type (WT) strain. A muscle-specific GAL4, Dmef2 (BL27390); a pan-neuronal GAL4 for expression in pupal and adult stages, $n S y b^{30589}$ (BL39171; Pfeiffer et al., 2008); a glutamatergic neuron GAL4, OK371 (BL26160); and the orai ${ }^{3}$ mutant strains (BL17538) were obtained from the Bloomington Drosophila Stock Center (BDSC; Department of Biology, Indiana University). The aminergic GAL4s, $D d c$, $T H$, and TRH have been published previously and were obtained from their published sources (Lundell and Hirsh, 1994; Friggi-Grelin et al., 2003; Huser et al., 2012). The THGAL4, UASH2BmRFP, and THGAL4, UASmGFP strains were generated by recombining UASH2BmRFP (Langevin et al., 2005) and UASmGFP (Lee et al., 1999) with THGAL4 respectively. The UAS dOrai strain (referred to as $\mathrm{COrai}^{+}$) was generated by cloning of the publicly available full-length cDNA (RE30427) of dOrai (corresponding to the B transcript), in the pUAST vector (Venkiteswaran and Hasan, 2009), followed by microinjection to obtain a transgenic fly strain. A dOrai cDNA with the E180A mutation was a gift from Shenyuan L. Zhang (Yeromin et al., 2006). The cDNA for $\mathrm{OOrai}^{\text {E180A }}$ was subcloned in the pUASTattb vector between EcoRI and XhoI sites and microinjected in embryos to generate the UASE180A strain. A GAL80 ${ }^{\text {ts }}$ strain with two insertions on the second chromosome was generated by Albert Chiang (National Centre for Biological Sciences, Bangalore, India). TH subdomain GAL4 strains (THD1, THD', THD4, and THC') were kindly provided by Mark Wu, Johns Hopkins University, Baltimore, Maryland (Liu et al., 2012). UASRNAi strains dsdSTIM (v47073), dsDTH (v108879), $d s d D A T$ (v12082), and $d s d O r a i(\mathrm{v} 12221)$ were obtained from the Vienna Drosophila Resource Center (Campus Science Support Facilities, Vienna, Austria). dsitpr (1063-R2) was obtained from the National Institute of Genetics (Shizuoka, Japan). UAS effector strain for Cacl (BL8581) was obtained from the BDSC and for DTHg and DTH1 from Serge Birman (Friggi-Grelin et al., 2003). UASdicer strains were obtained from the BDSC.

Flight assays and electrophysiological recordings. Single fly tests were performed with flies of either sex for measuring the time of flight bouts during air puff-stimulated tethered flight were performed as described previously (Agrawal et al., 2013). Flies from individual genotypes were collected 3-4 d after eclosion and tested for flight for $\leq 30 \mathrm{~s}$. All videos were recorded for $30 \mathrm{~s}$ after flight initiation at 24 frames per second. The $30 \mathrm{~s}$ time period was based on our observation that flies exhibiting $30 \mathrm{~s}$ flight bouts usually continued flying for longer periods. Significant differences between flight performances of various genotypes were obtained by creating box plots of flight times, obtained from $\geq 30$ flies of each genotype, using Origin 8 software (Micro Cal). Flight-duration box plots show the range of flight times of the genotypes tested, where the box represents 25 and $75 \%$ of the data points, the horizontal line is the median, and the small square-shaped solid box represents the mean. Flight times of individual flies appear as diamonds. Electrophysiological recordings for flight were obtained by inserting a sharpened tungsten electrode in fiber "a" of the indirect dorsal longitudinal muscles (DLMs). The recordings were performed as described previously (Banerjee et al., 2004; Agrawal et al., 2013). Average durations of DLM firing from $\geq 15$ individuals are shown as histograms and diamonds represent the duration of DLM firing recorded from individual flies.

Climbing assays. Climbing assay was performed using a glass cylinder with a diameter of $2.5 \mathrm{~cm}$. Flies of the appropriate genotype (3-4 d after eclosion) were dropped in the cylinder in batches of 10. A gentle tap was given three times so that all the flies reach the bottom of the cylinder. Then the number of flies that crossed an $8 \mathrm{~cm}$ mark in $12 \mathrm{~s}$ were counted manually. Each batch of flies was tested three times. Means and SEMs were calculated from $\geq 3$ batches of 10 flies each using Origin 8 software (Micro Cal).

FACS of dopaminergic neurons. Approximately 15-20 CNSs were dissected in cold Schneider's medium (\#21720-024, Life Technologies) from $48 \mathrm{~h}$ pupae of each genotype. The dissected CNSs were incubated in cysteine-activated papain $(50 \mathrm{U} / \mathrm{ml})$ for $30 \mathrm{~min}$ at room temperature $\left(25^{\circ} \mathrm{C}\right)$ and then spun at $3000 \mathrm{rpm}$ for $3 \mathrm{~min}$. After discarding the supernatant, the brains were resuspended in cold Schneider's medium and gently triturated to obtain single-cell suspensions. This suspension was passed through a $40 \mu \mathrm{m}$ mesh filter and kept on ice until sorting. Flow cytometry was performed on a FACS Aria cell sorter (BD Biosciences) equipped with a $488 \mathrm{~nm}$ laser to detect GFP. The threshold for GFPpositive cells was set using dissociated neurons from a non-GFPexpressing wild-type strain, Canton $S$. The same gating parameters were used to sort all genotypes in the experiment. GFP-positive and GFPnegative cells $(\sim 10,000$ each $)$ were collected directly in TRIzol Reagent (Life Technologies) for further processing. Three independent sets of FACS-sorted neurons were used for RNA isolation.

$R N A$ isolation, semiquantitative $P C R$, and $q P C R$. For RNA isolation, the CNS of either third-instar larvae, pupae of specific ages, or adult flies were dissected and homogenized in TRIzol Reagent (Life Technologies) and processed further according to the manufacturer's protocol. RNA integrity was determined on a $1 \%$ Tris-acetate-EDTA agarose gel. At least three independently isolated RNA samples were obtained for each genotype. Total RNA ( $\sim 500 \mathrm{ng})$ was treated with $0.5 \mathrm{U}$ of DNase I (amplification grade) in a reaction mixture $(22.1 \mu \mathrm{l})$ containing $1 \mathrm{~mm}$ DTT and $20 \mathrm{U}$ of RNase inhibitor. The reaction mixture was kept at $37^{\circ} \mathrm{C}$ for 30 min followed by heat inactivation at $70^{\circ} \mathrm{C}$ for $10 \mathrm{~min}$. To this, $200 \mathrm{U}$ of Moloney murine leukemia virus reverse transcriptase, $50 \mu \mathrm{M}$ random hexamers, and $1 \mathrm{~mm}$ deoxyribonucleotide triphosphate were added in a 
A

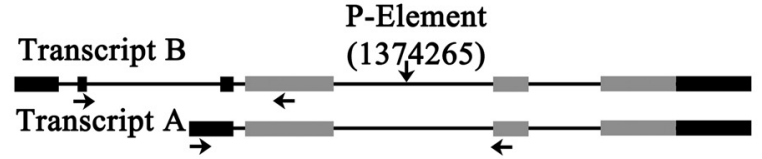

B

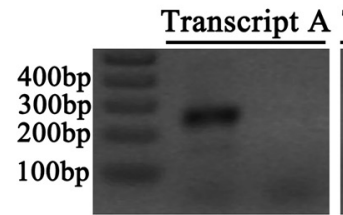

C
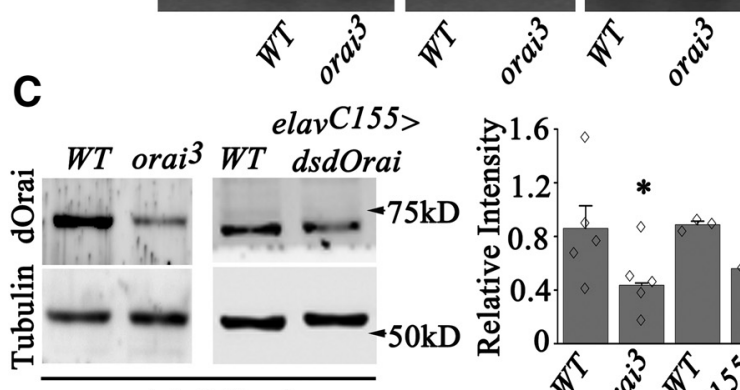

Adult Head

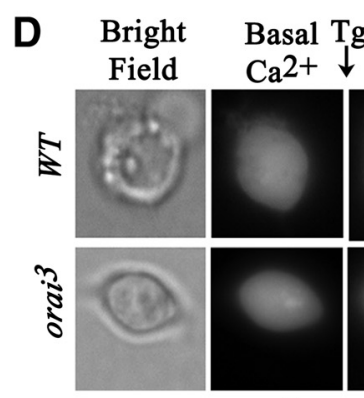

Os

E
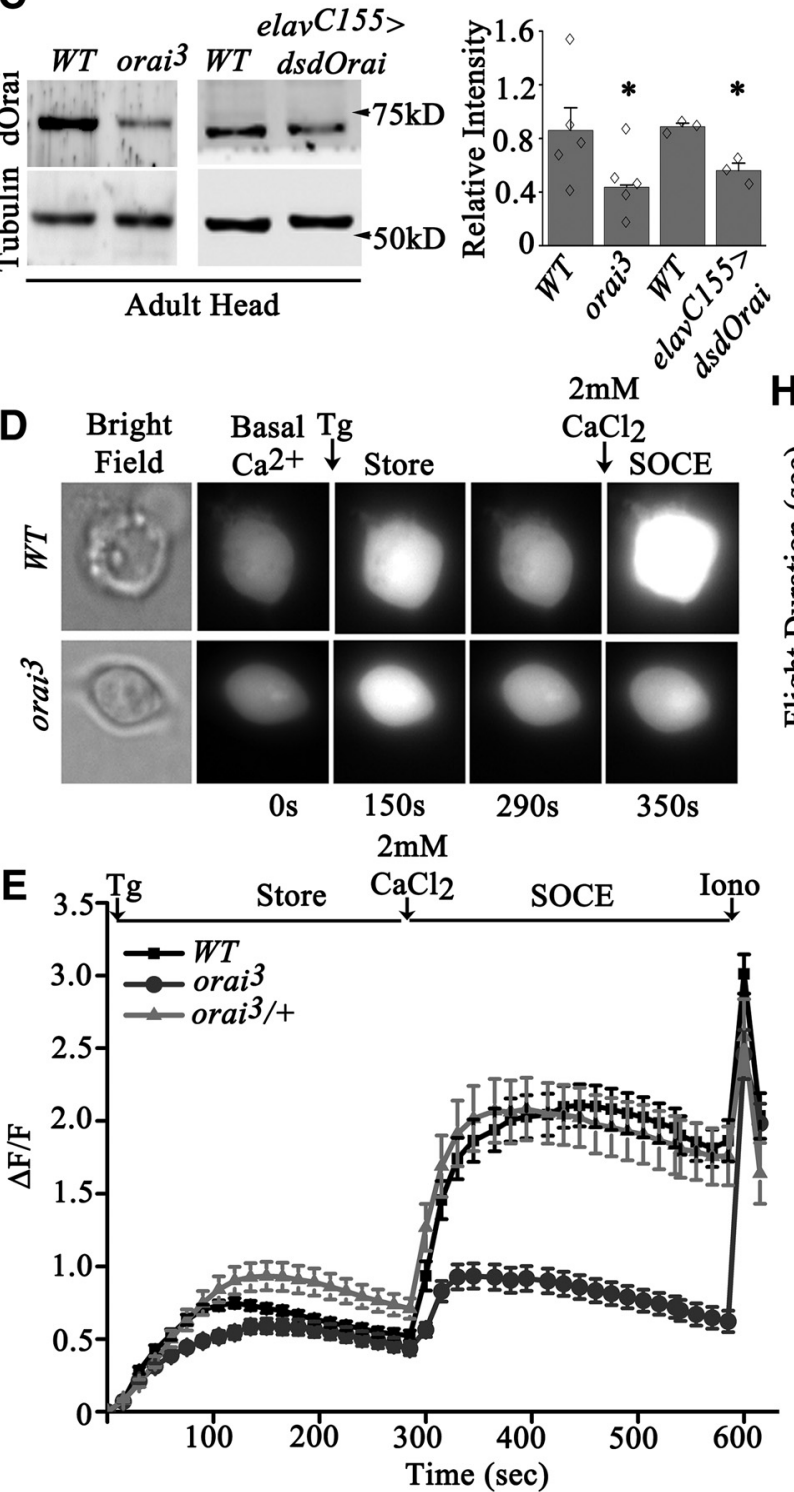

$2 \mathrm{mM}$

$\mathrm{CaCl}_{2}$

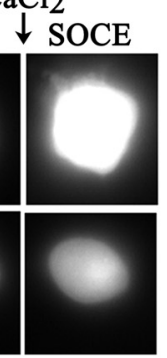

$350 \mathrm{~s}$

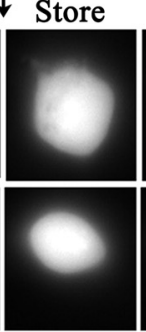

$150 \mathrm{~s}$ 290s

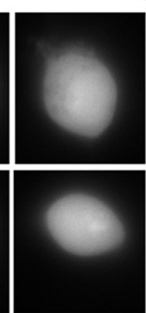

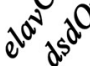

F

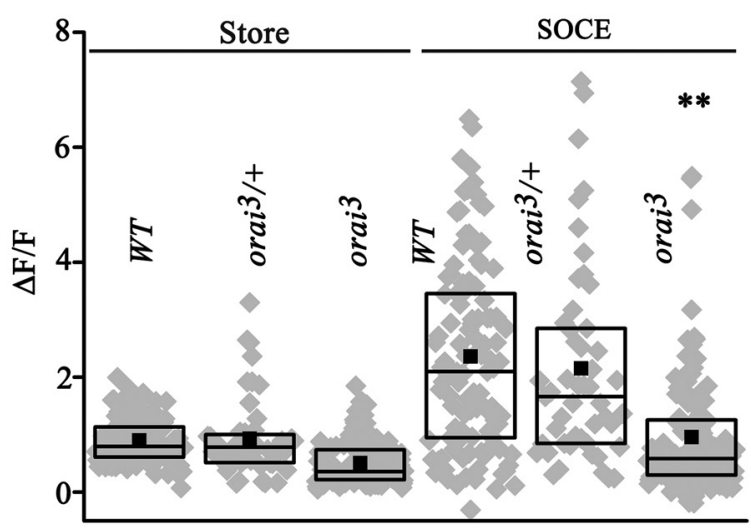

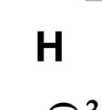
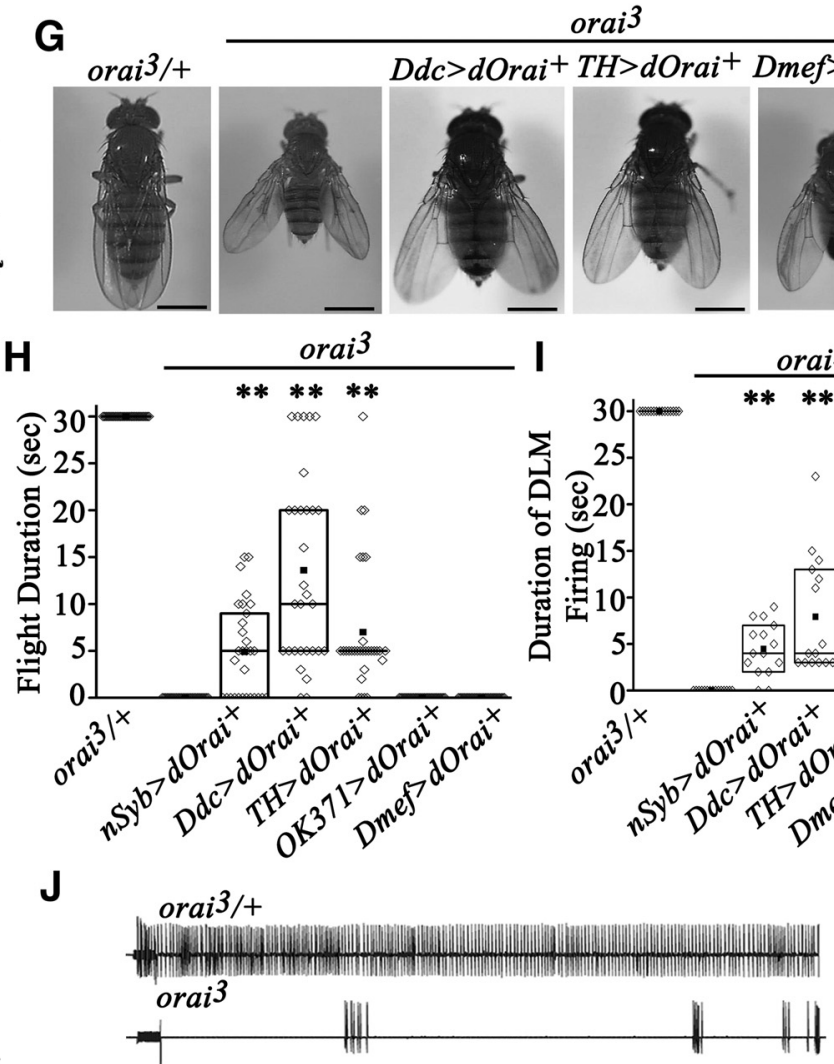

$\mathrm{Ddc}>\mathrm{dOrai}^{+}$

orai 3

J

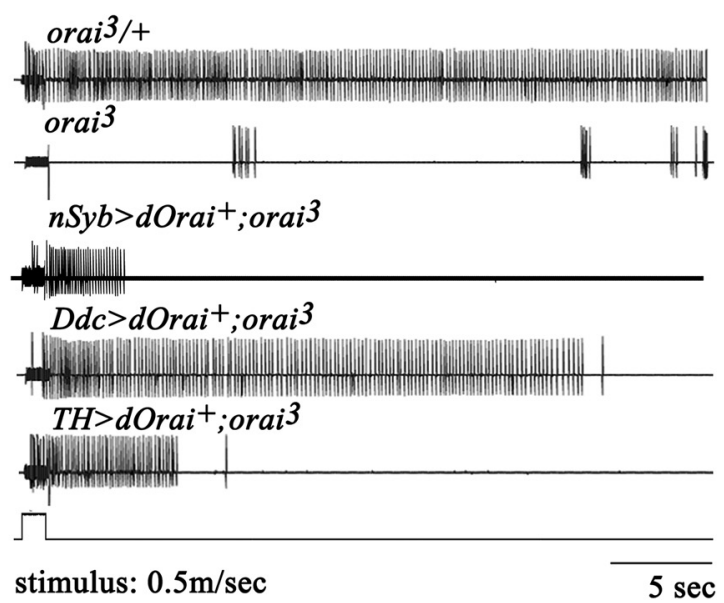

Figure 1. The orai ${ }^{3}$ mutant exhibits reduced SOCE in primary neurons and flight deficits in adult Drosophila. $A$, Schematic representation of transcript $A$ and $B$ of $d O$ rai. The thin line represents introns, the thick dark lines represent noncoding regions of the exon, and the light gray lines represent coding regions of the transcripts. The P element is inserted at position 1374265 (Cuttell et al., 2008). The arrows represent positions of primers used to perform semiquantitative PCR. $B$, Image of an agarose gel with RT-PCR products identifying transcripts $A$ and $B$ in RNA isolated from adult heads of the indicated genotypes. $C$, A representative Western blot of protein lysates from heads of orai ${ }^{3}$ mutants, elav ${ }^{\mathrm{C} 155}>d s d 0$ rai, and a wild-type strain (CS), probed with the d0rai antibody. The relative intensity of dOrai bands normalized to tubulin from $\geq 3$ such blots was quantified with ImageJ (1.48V Java 1.6.0_20) and is shown in the bar graph. Diamonds represent relative intensity of dOrai bands from individual blots. The level of d0rai in lysates from orai ${ }^{3}$ and elav ${ }^{(155}>d s d$ Orai heads was significantly different compared with wild type $\left(N \geq 3 ;{ }^{*} P_{t \text { test }}<0.05\right)$. A UASDicer transgene was included to enhance the effect of $d s d O r a i$. $\boldsymbol{D}$, Images of primary neurons loaded with the calcium reporter dye Fluo-4 to measure store $\mathrm{Ca}^{2+}$ release after thapsigargin (Tg) treatment and SOCE upon addition of calcium $\left(\mathrm{CaCl}_{2}\right)$. E, Average traces of store $\mathrm{Ca}^{2+}$ release and SOCE in primary neurons from the indicated genotypes $(N \geq 100)$. Iono, lonomycin. $\boldsymbol{F}$, The range of maximum peak intensity is lowest in orai ${ }^{3}$ compared with the indicated genotypes shown as box plots. The midline represents the median and the solid box represents the mean of the (Figure legend continues.) 
final volume of $25 \mu \mathrm{l}$ for cDNA synthesis. The reaction mixture was kept at $25^{\circ} \mathrm{C}$ for $10 \mathrm{~min}$, then $42^{\circ} \mathrm{C}$ for $60 \mathrm{~min}$, and finally heat inactivated at $70^{\circ} \mathrm{C}$ for $10 \mathrm{~min}$. All reagents were from Invitrogen (Life Technologies). RT-PCR was performed in a reaction mixture of $25 \mu \mathrm{l}$ with $1 \mu \mathrm{l}$ of the cDNA.

Real-time quantitative PCRs (qPCR) were performed on an ABI 7500 Fast machine (Applied Biosystems) operated with ABI 7500 software version 2 in a total volume of $10 \mu \mathrm{l}$ using the KAPA SYBR FAST qPCR kit (\#KK4601, Kapa Biosystems). rp49 was used as an internal control. In the developmental profile of $T H, A c t 5 c$ was used as an additional internal control. A melt curve was performed at the end of the reaction to ensure a single peak. Sequences of the primers used ( $5^{\prime}$ to $\left.3^{\prime}\right)$ are as follows: $r p 49$ : forward, CGGATCGATATGCTAAGCTGT; reverse, GCGCTTGTTCGATCCGTA; transcript A: forward, GCACCACAGCAACAGTCCCA; reverse, GCATCCAGCAAGACGTCCG; transcript $B$ : forward, GTGTTGGTGTTATGATGCGATGG; reverse, GTCGTGGTGAAGGTCGTG C; $A c t 5 c$ : forward, GTCCACCTTCCAGCAGATG; reverse, CCTCCTCCAGCAGAATCAAG; $T H$ : forward, GTTGCAGCAGCCCAAAAGAAC; reverse, GAGACCGTAATCATTTGCCTTGC; $d$ Cacl: forward, TTTCG TTGTAGTTACGGGAGCC; reverse, ATTTGCAGTAAAGGTGCCATC G; dSlo: forward, GCGGTGAACTGAAAAGAGAAC; reverse, CAAAT CGGGTGGTTTACGATGG; dDAT: forward, CATGGCCCACACACT GGT; reverse, GATCTTGGGAAACTCGTCGCTC; dOrai: forward, GAGATAGCCATCCTGTGCTGG; reverse, CGGATGCCCGAGAC TGTC.

The fold change of gene expression in the mutant relative to wild type was determined by the comparative $\Delta \Delta C_{\mathrm{t}}$ method (Lorentzos et al., 2003). Fold change equals $2^{-\Delta \Delta C t}$, where $\Delta \Delta C_{t}$ equals $\left(C_{\mathrm{t}(\text { target gene })}-C_{\mathrm{t}(\mathrm{rp} 49)}\right)_{\text {mutant } 2}-\left(C_{\mathrm{t}(\text { target gene })}-C_{\mathrm{t}(\mathrm{rp} 49)}\right)_{\text {wild type }}$.

Immunohistochemistry. Immunostaining of adult Drosophila brains was performed as described previously (Sadaf et al., 2015). The following primary antibodies were used: rabbit anti-GFP antibody $(1: 10,000$; A6455, Life Technologies), mouse anti-TH antibody (1:40; Immunostar), mouse anti-bruchpilot (anti-brp) antibody (1:50; kindly provided by Eric Buchner, Univerity of Wuerzburg, Germany). Secondary antibodies were used at a dilution of 1:400 as follows: anti-rabbit Alexa Fluor 488 (\#A1108, Life Technologies) and anti-mouse Alexa Fluor 568 (\#A1104, Life Technologies). Confocal images were obtained on the Olympus Confocal FV1000 microscope (Olympus). For comparative studies of control and experimental samples, identical confocal settings were used and experiments were performed in parallel on the same day. Image visualization and analysis was done using an FV10-ASW 1.3 viewer (Olympus).

Primary neuronal cultures from Drosophila third-instar larvae and pupae. The culturing of Drosophila primary neurons was performed as described previously (Venkiteswaran and Hasan, 2009; Agrawal et al., 2010; Chakraborty and Hasan, 2012) with minor modifications. Dissociated neurons obtained from either third-instar larval or 32-48 h pupal brains were cultured in $200 \mu \mathrm{l}$ of DMEM/nutrient mixture F12 supplemented with $50 \mathrm{U} / \mathrm{ml}$ Pen Strep (Life Technologies) and 10 $\mu \mathrm{g} / \mathrm{ml}$ amphotericin B. All reagents for culturing primary neurons

(Figure legend continued.) distribution ( $N \geq 100 ;{ }^{* *} p<0.001$, Mann-Whitney test). $\mathbf{G}$, Images of adult Drosophila of the indicated genotypes. The orai ${ }^{3}$ mutant exhibits expanded wing posture and reduced body size compared with controls. Scale bars, $0.5 \mathrm{~mm}$. $\boldsymbol{H}$, Flight time obtained in response to an air puff. The box represents the data range from 25 to $75 \%$ and diamonds represent the flight time of individual flies. The mean (solid square box) and median (solid horizontal line) of the data are shown $\left(N=30\right.$; ${ }^{* *} p<0.001$, Mann-Whitney test). orai ${ }^{3}$ homozygotes are flightless and can be partially rescued by overexpression of $\mathrm{dOrai}^{+}$in neurons. I, Duration of DLM firing, calculated from electrophysiological recordings (shown in $J$ ), are reduced in orai mutants. Overexpression of $\mathrm{dOrai}^{+}$in either pan-neuronal (nSyb), aminergic $(D d c)$, or dopaminergic $(T H)$ neuronal domains can partially rescue the duration of DLM firings $\left(N=15 ;{ }^{* *} p<0.001\right.$ Mann-Whitney test).J, Representative electrophysiological recordings obtained from the dorsal longitudinal indirect flight muscles (DLMs) of tethered flies during an air puff-simulated flight bout. Normal flight patterns were absent in orai ${ }^{3}$ mutants. Panneuronal expression of $\mathrm{dOrai}^{+}$as well as overexpression of $\mathrm{dOrai}^{+}$in either aminergic or dopaminergic neuronal domains could partially rescue the flight patterns. were obtained from Life Technologies. Cultured neurons were kept at $25^{\circ} \mathrm{C}$ with $5 \% \mathrm{CO}_{2}$ for $14-16 \mathrm{~h}$ before imaging. This protocol for culturing primary neurons does not support the growth of glial cells, which remain at $<1 \%$ of the total cell population (Wu et al., 1983).

Calcium imaging in primary neurons. After $14-16 \mathrm{~h}$ of growth, cells were washed with Drosophila M1 media (Agrawal et al., 2010) twice and loaded with $2.5 \mu \mathrm{M}$ fluo-4 acetoxymethyl ester (AM; Life Technologies) plus $0.002 \%$ Pluronic-F127 (Sigma-Aldrich) in M1 medium by incubation for $30 \mathrm{~min}$ in the dark at room temperature. After dye loading, the cells were washed with M1 three times and covered with $100 \mu \mathrm{l}$ of calcium-free M1 containing $0.5 \mathrm{~mm}$ EGTA. The cells were imaged within $40 \mathrm{~min}$ of dye loading. Imaging and quantification were performed as described previously (Agrawal et al., 2010). Briefly, store depletion was achieved by adding $10 \mu \mathrm{M}$ thapsigargin (Life Technologies; Venkiteswaran and Hasan, 2009) and SOCE was measured by the addition of $2 \mathrm{mM} \mathrm{CaCl}_{2}$. Ionomycin $(10 \mu \mathrm{M}$; Calbiochem-407950, Merck Millipore) was added after 40 frames or $600 \mathrm{~s}$ so as to confirm that Fluo-4 levels were not at saturation values during the experiment. Addition of thapsigargin, $\mathrm{CaCl}_{2}$, and ionomycin was done manually at the indicated time points. Images were acquired automatically every $15 \mathrm{~s}$ over a period of $11 \mathrm{~min}$.

Data analysis. For measuring changes in fluorescence, images were processed using the ImagePro plus software, V1.33 (Media Cybernetics). Fluorescence intensity at various time points was determined. The data were plotted with Origin 8.0 software as follows: $\Delta F / F=$ $\left(F_{\mathrm{t}}-F_{\text {basal }}\right) / F_{\text {basal }}$ for each time point where $F_{\mathrm{t}}$ is the fluorescence at the time point and $F_{\text {basal }}$ is the fluorescence of the cell when starting the experiment. Peak values of $\Delta F / F$ were obtained for every cell that responded and a box chart representing the data spread was plotted. The rectangular boxes represent the spread of data points between 25 and $75 \%$ of cells and the horizontal line is the median. Significant differences between multiple groups of data were analyzed by the Mann-Whitney test for nonparametric datasets and significant differences were taken at $p<0.05$.

Generation of an antibody to dOrai in rats. The $\mathrm{OOrai}^{+}$cDNA was subcloned in the pGEX6P1 expression vector (GE Healthcare Life Sciences) between restriction sites for EcoRI and XhoI, so as to tag the protein with GST at the $\mathrm{N}$ terminus. The Escherichia coli strain BL21 codon $^{+}$was transformed with the tagged $\mathrm{dOrai}^{+}$cDNA clone. After overnight induction of the protein at $18^{\circ} \mathrm{C}$ and $180 \mathrm{rpm}$ by addition of $0.3 \%$ isopropyl- $\beta$-D-thiogalactoside, cells were harvested from $5 \mathrm{~L}$ of culture, washed with PBS, and resuspended in $100 \mathrm{ml}$ of lysis buffer (25 mm HEPES, pH 7.4, $250 \mathrm{~mm} \mathrm{NaCl}, 5 \%$ glycerol, 1 mм DTT, 1\% Triton X-100, $1 \mathrm{~mm}$ PMSF, and protease inhibiter mixture from Roche). Harvested cells were sonicated and centrifuged at 12,000 rpm at $4^{\circ} \mathrm{C}$ for $30 \mathrm{~min}$. The filtered supernatant $(100 \mathrm{ml})$ was loaded on a GST column ( $5 \mathrm{ml}$; GE Healthcare). After washing with five column volumes of washing buffer A (25 mM HEPES, pH 7.4, $250 \mathrm{~mm} \mathrm{NaCl}$, $5 \%$ glycerol, $1 \mathrm{~mm}$ DTT, $1 \%$ Triton X-100) and five column volumes of washing buffer B (25 mm HEPES, pH 7.4, $1 \mathrm{~m} \mathrm{NaCl}, 5 \%$ glycerol, 1 mм DTT, 1\% Triton X-100), the GST-tagged protein was eluted from the column by addition of $15 \mathrm{ml}$ of $15 \mathrm{~mm}$ reduced glutathione (Sigma-Aldrich). The eluted protein was concentrated to $2 \mathrm{ml}$ with a $100 \mathrm{kDa}$ centricon filter (Amicon Ultra, Millipore) and further purified on a Sephadex-200 gel filtration column (GE Healthcare). The fraction corresponding to tagged dOrai $(75 \mathrm{kDa})$ was reconcentrated in a $100 \mathrm{kDa}$ centricon filter and $3 \mathrm{mg}$ of purified protein was obtained. The purified protein was given to a company (Abexosome Biosciences) for generating an antibody in rats.

Western blots. Larval, pupal, and adult CNSs of appropriate genotypes were dissected in cold PBS. Between 5 and 10 brains were homogenized in $50 \mu \mathrm{l}$ of homogenizing buffer ( 25 mм HEPES, pH 7.4, $150 \mathrm{~mm} \mathrm{NaCl}, 5 \%$ glycerol, $1 \mathrm{~mm}$ DTT, $1 \%$ Triton X-100, and $1 \mathrm{~mm}$ PMSF) and $10-15 \mu \mathrm{l}$ of the homogenate was run on an $8 \%$ SDSpolyacrylamide gel. The protein was transferred to a nitrocellulose membrane by standard protocols and the membrane was incubated in the primary antibody overnight at $4^{\circ} \mathrm{C}$. Primary antibodies were used at the following dilutions: rat anti-dOrai, 1:1500; rabbit anti-TH (MAB318, Merck Millipore), 1:1000; mouse anti- $\beta$-tubulin mono- 
A

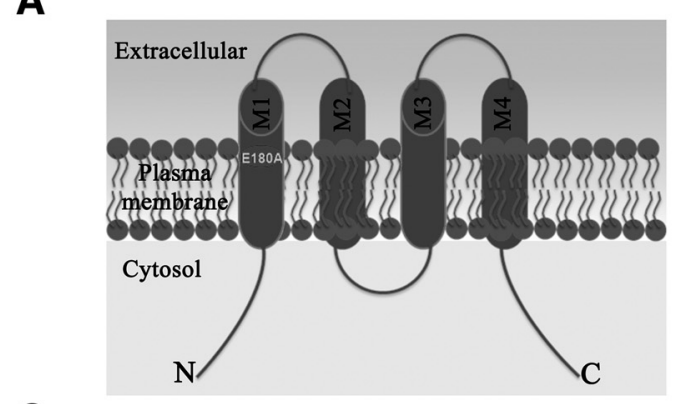

c

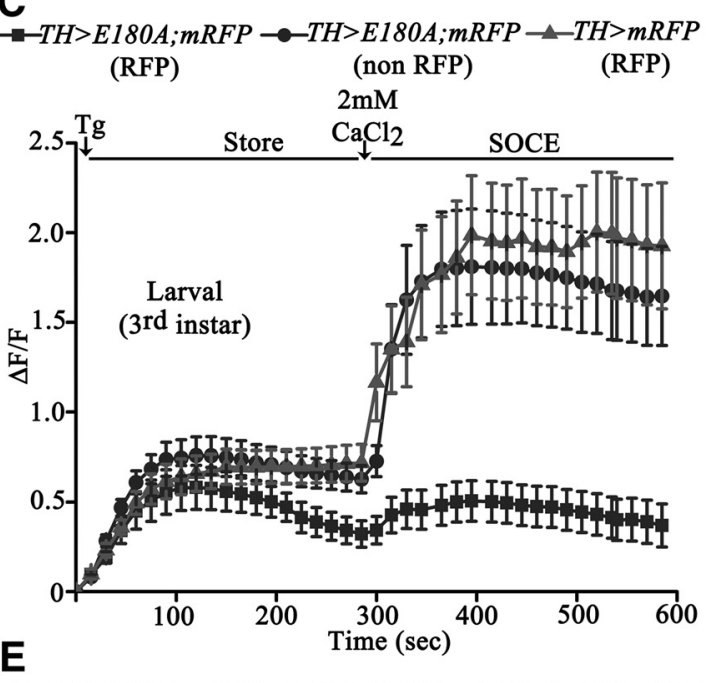

E

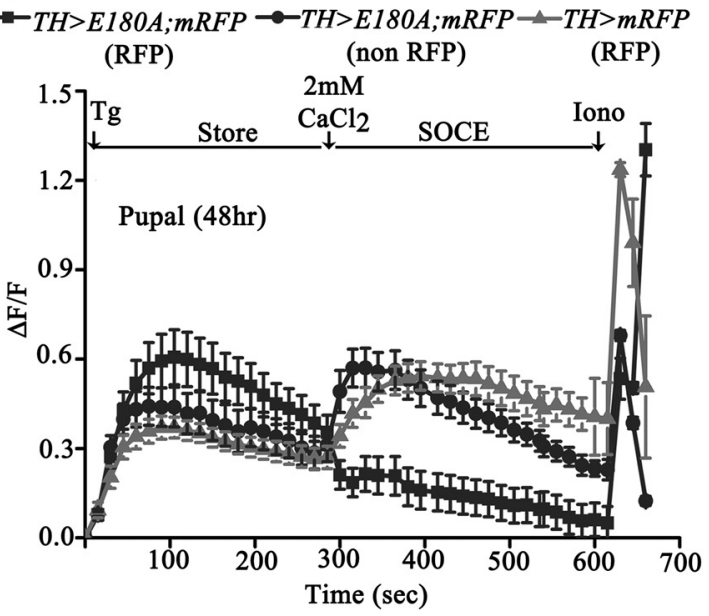

B

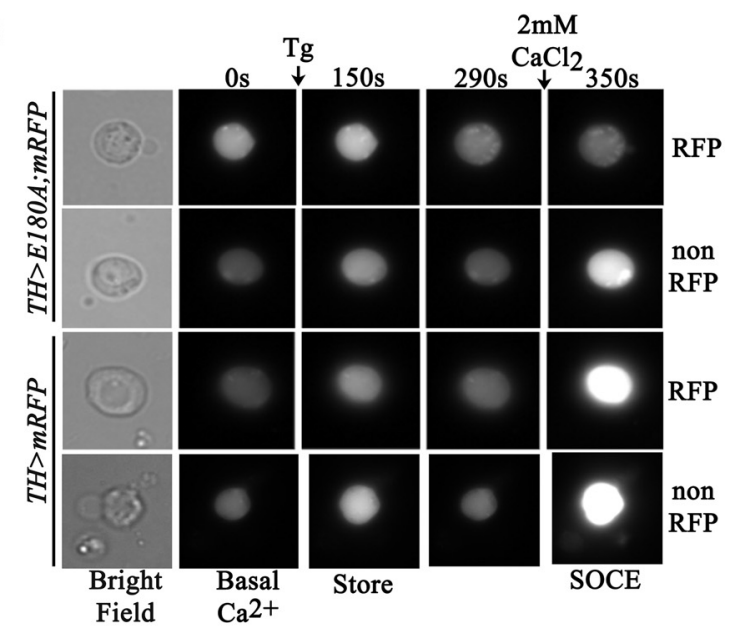

D

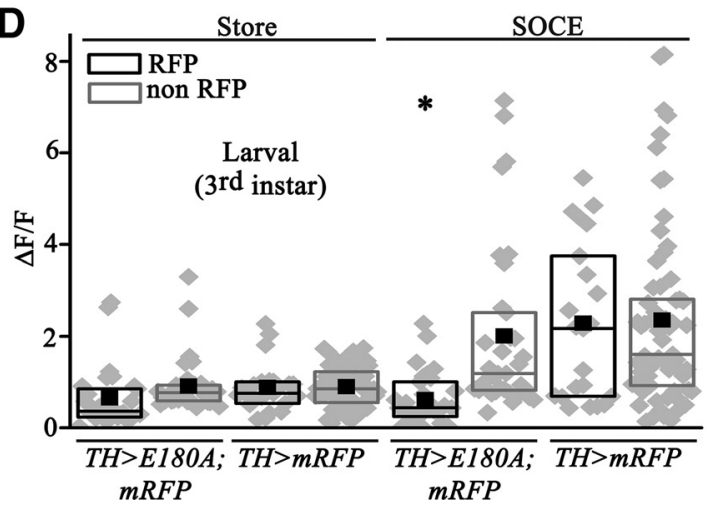

$\mathbf{F}$

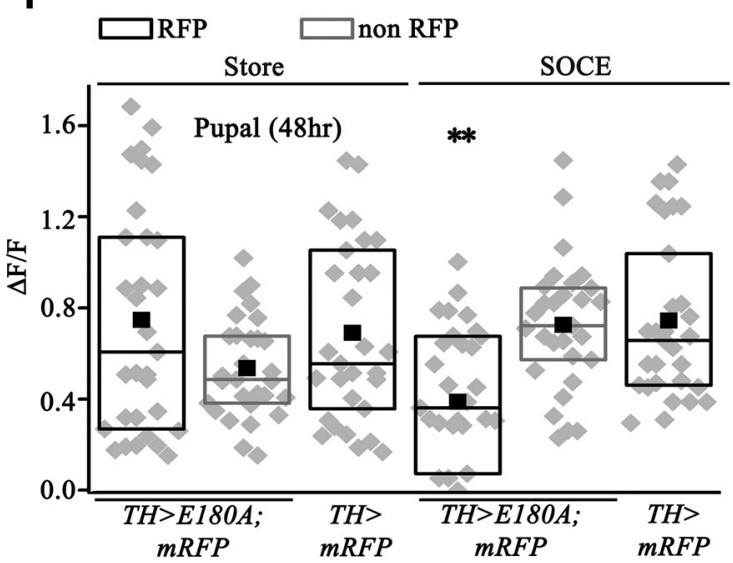

Figure 2. Overexpression of a dominant-negative dOrai transgene, $\mathrm{dOrai}{ }^{\mathrm{E} 180 \mathrm{~A}}$, in dopaminergic neurons reduces SOCE. $A$, Schematic representation of a monomer of the d0rai channel. The single point mutation E180A (glutamic acid to alanine at position 180) is present in the first transmembrane domain. $\boldsymbol{B}$, Representative images of cultured single neurons from third-instar larval brains of the indicated genotypes. Overexpression of E180A in dopaminergic neurons (RFP cells in TH $>E 180 \mathrm{~A} ; m R F P$ ) leads to reduced SOCE. C, The traces show mean store release and SOCE. The first peak represents passive store $\mathrm{Ca}^{2+}$ release upon addition of thapsigargin, whereas the second peak represents SOCE upon addition of extracellular $\mathrm{Ca}^{2+}(N \geq 30)$. D, The range of maximum $\Delta F / F$ values for individual cells from the indicated genotypes are shown as box plots. The midline represents the median and the solid box represents the mean of the distribution $\left(N \geq 30\right.$; ${ }^{*} p<0.01$ Mann-Whitney test). $E$, Values of $\triangle F / F \pm$ SEM calculated from primary neurons of $48 \mathrm{~h}$ pupae of the indicated genotypes $(N \geq 30)$. SOCE was reduced upon expression of $E 180 A$ in pupal dopaminergic neurons (RFP cells in TH>E180A; $m R F P$ ). $\boldsymbol{F}$, Box plots with distribution of peak $\triangle F / F$ values from individual cells after thapsigargin and $\mathrm{Ca}^{2+}$ addition. SOCE was reduced in E180A-expressing pupal dopaminergic neurons (RFP cells in TH $>E 180 A ; m R F P$ ) compared with controls (non-RFP cells in TH $>E 180 A ; m R F P$ or RFP cells in $T H>E 180 A ; N \geq 30$, ${ }^{* *} p<0.001$, Mann-Whitney test)

clonal (E7; Developmental Studies Hybridoma Bank, University of Iowa, Iowa), 1:5000. Secondary antibodies conjugated with horseradish peroxidase were used at dilution of 1:3000 (anti-mouse HRP; 7076S, Cell Signaling Technology), 1:3000 (anti-rabbit HRP; 32260, Thermo Scientific), and 1:10,000 (anti-rat HRP; 012030003, Jackson Immuno Research). The protein was detected on the blot by a chemiluminiscent detection solution from Thermo Scientific.

\section{Results}

Flight deficits and reduced SOCE are observed in the orai $^{3}$ mutant

The Drosophila Orai gene dOrai encodes two major transcripts, A and $\mathrm{B}$, which can both be translated to give the full-length dOrai protein (Fig. 1A). We characterized neuronal phenotypes of a 
A

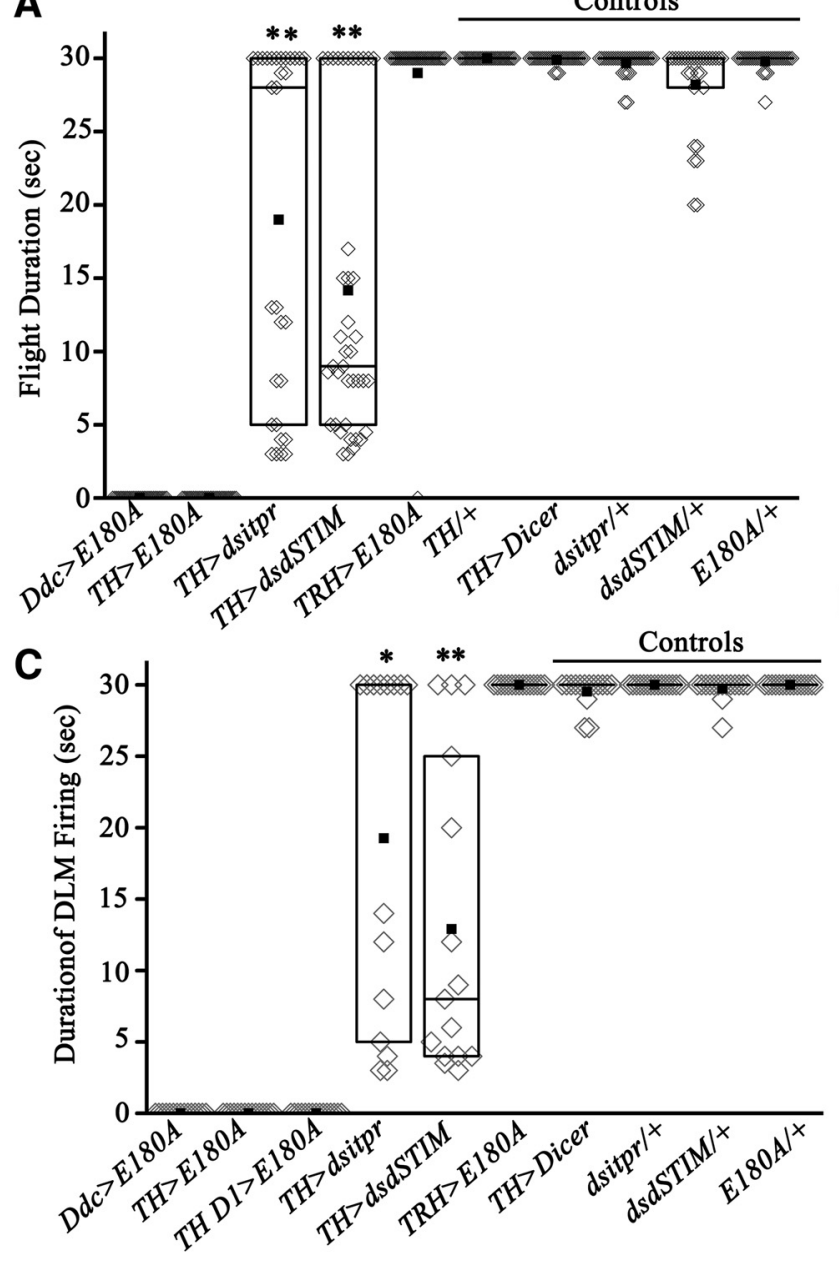

B

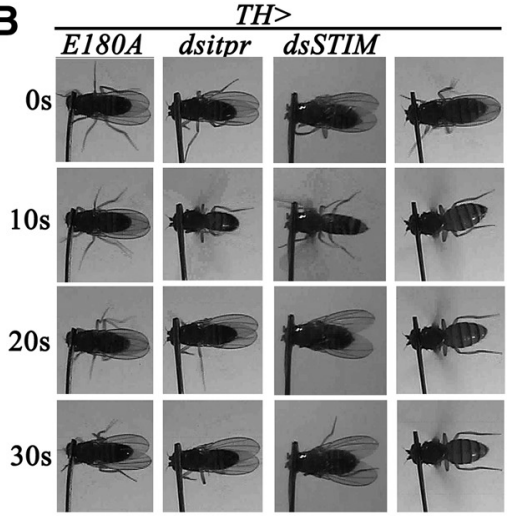

D

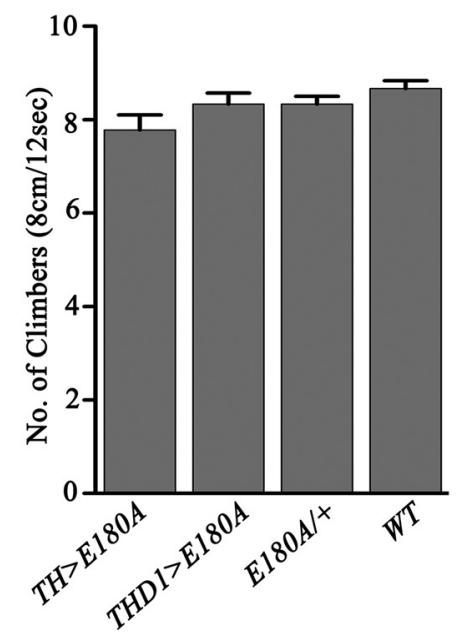

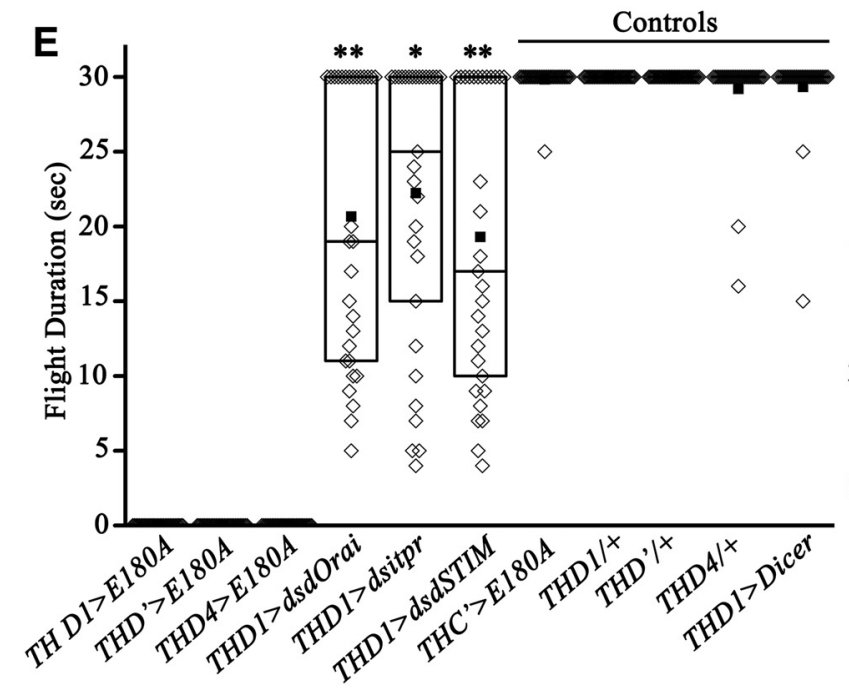

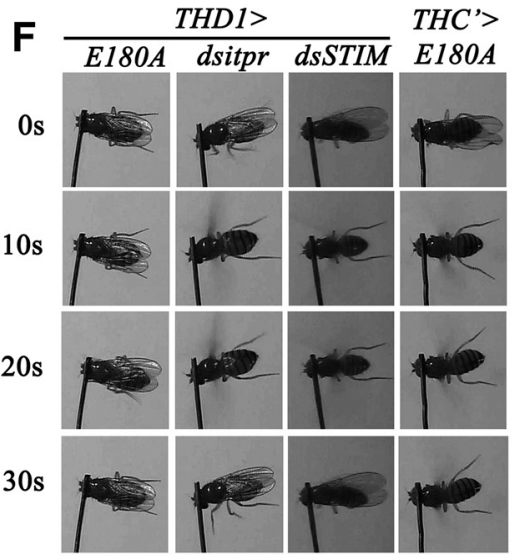

Figure 3. SOCE is required in dopaminergic neurons for flight. $\boldsymbol{A}$, Flight time of flies of the indicated genotypes ( $N \geq 30,{ }^{* *} p<0.001$ Mann-Whitney test). $\boldsymbol{B}$, Snapshots of flight videos at the indicated times during air puff-stimulated flight bouts. $C$, Average duration of DLM firing trains. DLM recordings from flies of the indicated genotypes were obtained during an air puff-induced flight bout $\left(N=15 ;{ }^{*} p<0.01,{ }^{* *} p<0.001\right.$, Mann-Whitney test). $\boldsymbol{D}$, Average number of flies of the indicated genotypes that passed the climbing test. No significant difference was observed between various genotypes tested. $\boldsymbol{E}$, Flight time of flies of indicated genotypes $\left(N \geq 30 ;{ }^{*} p<0.01,{ }^{* *} p<0.001\right.$, Mann-Whitney test). $\boldsymbol{F}$, Snapshots of flight videos at the indicated time intervals of air puff-stimulated flight bouts. THD1GAL4-driven overexpression of E180A resulted in complete loss of flight (extreme left). A UASDicer transgene was included with all the RNAi strains tested.

hypomorphic allele of dOrai, olf186- $F^{E Y 09167}$ (Cuttell et al., 2008), henceforth referred to as orai ${ }^{3}$, which is a publicly available P-element insertion line. The position of the P-element insertion within the fourth intron (2R-1374265; Fig. 1A) of dOrai was also confirmed by sequencing. Transcript A was significantly reduced in the CNS of $\mathrm{orai}^{3} / \mathrm{orai}^{3}$ adults, whereas expression of transcript B was not affected significantly (Fig. 1B). These data suggest complex regulation of the dOrai gene in the nervous system, which 
Table 1. PPM3 and PPL1 clusters and the a-a' dopaminergic neuron pair are marked by TH, THD1, THD', and THD4 GAL4s, but not by THC'GAL4

\begin{tabular}{|c|c|c|c|c|c|c|}
\hline & $\mathrm{TH}$ & THD1 & THD4 & $T H D^{\prime}$ & $T H C^{\prime}$ & $\overline{T H D 1>E 180 A}$ \\
\hline \multicolumn{7}{|c|}{ TH-positive neurons per hemisphere $(n=12)$} \\
\hline PAM & $11-13$ & $0-2$ & $1-2$ & 0 & $0-1$ & $0-1$ \\
\hline Protocerebral anterior lateral & $4-7$ & 4 & 0 & 0 & $3-4$ & 4 \\
\hline PPM1 & 1 & 0 & 0 & 0 & 0 & 0 \\
\hline PPM2 & $5-6$ & $2-3$ & $2-3$ & 0 & $5-6$ & $2-3$ \\
\hline PPM3 & $6-8$ & $6-7$ & $6-7$ & $6-7$ & 0 & $6-7$ \\
\hline PPM4 & $0-1$ & 0 & 0 & 0 & 0 & 0 \\
\hline PPL1 & 12 & $10-11$ & $7-9$ & 10 & 0 & $10-11$ \\
\hline PPL2 & $7-8$ & $4-5$ & 0 & $5-6$ & $5-6$ & $3-5$ \\
\hline PPL3 & $0-1$ & 0 & 0 & 0 & 0 & 0 \\
\hline PPL4 & $0-1$ & 0 & 0 & 0 & 0 & 0 \\
\hline Subesophageal ventral posterior & 1 & 0 & 0 & 0 & 1 & 0 \\
\hline Ventral unpaired medial 1 & 1 & 1 & 0 & 0 & 1 & 1 \\
\hline Ventral unpaired medial 2 & 2 & 2 & 0 & 0 & 2 & 2 \\
\hline \multicolumn{7}{|c|}{ TH-positive neurons in ventral ganglia $(n=6)$} \\
\hline $\mathrm{T} 1$ & $5-6$ & $2-3$ & 0 & 0 & 5 & $2-3$ \\
\hline \multicolumn{7}{|l|}{$\mathrm{T} 2$} \\
\hline$a-a^{\prime}$ & 2 & 2 & 2 & 2 & 0 & 2 \\
\hline b-b', ventral unpaired medial & $2-3$ & 0 & 0 & 0 & 3 & 0 \\
\hline $\mathrm{T} 3$ & $6-7$ & $0-1$ & 0 & 2 & 0 & $0-1$ \\
\hline Abdominal & $23-25$ & $23-25$ & 0 & $24-25$ & $4-5$ & $23-25$ \\
\hline
\end{tabular}

requires further characterization. Loss of transcript A was accompanied by reduction of dOrai level in the heads of $\mathrm{orai}^{3} / \mathrm{orai}^{3}$ adults to a similar extent as observed by RNAi knockdown of dOrai transcripts (Fig. 1C).

dOrai functions as a channel for extracellular calcium entry after depletion of intracellular calcium stores (SOCE; Feske et al., 2006; Vig et al., 2006). In primary neurons derived from homozygous ora $^{3}$ larval brains, a significant reduction in SOCE was observed, whereas release of store calcium, after inhibition of the sarco-ER $\mathrm{Ca}^{2+}$-ATPase by thapsigargin treatment, appeared similar to wild type (Fig. $1 D-F$ ). Neurons derived from orai ${ }^{3}$ heterozygotes exhibit normal store release and SOCE (Fig. 1D$F$ ). Thus both molecular and cellular phenotypes of orai $^{3} \mathrm{mu}^{-}$ tants confirm that it is a hypomorphic and recessive allele of dOrai.

To begin understanding the functional significance of reduced SOCE in neuronal function, adult viability of orai ${ }^{3}$ mutants was determined and found to be $\sim 20 \%$. Their lethality is very likely in the larval stages because all pupae eclosed as adults. Adults that eclosed appeared smaller than orai ${ }^{3}$ heterozygotes and had outspread wings (Fig. 1G). Previous results have established that knockdown of dOrai in Drosophila neurons reduces flight (Venkiteswaran and Hasan, 2009). In agreement with these published data, ora $^{3}$ mutant adults were flightless (Fig. 1H). Therefore, we tested flight in $\mathrm{orai}^{3}$ mutants after expression of a $\mathrm{COrai}^{+} \mathrm{cDNA}$ in either neurons or muscles with the help of the UAS-GAL4 system of tissuespecific and cell-specific expression (Brand and Perrimon, 1993). A small but significant rescue of the flight deficit in orai $^{3}$ mutants was obtained by neuronal expression of a $\mathrm{dOrai}^{+}$cDNA. Expression was driven by nsybGAL4 ${ }^{30589}$ with higher expression in pupae and adults compared with other neuronal drivers (Pfeiffer et al., 2008; Fig. 1H). However, orai ${ }^{3}$ flies with expression of $\mathrm{dOrai}^{+}$in muscles (Dmef; Fig. 1H) continue to be flightless, thus supporting a neuronal focus for dOrai function in flight. Previous findings also suggest that Drosophila flight requires intracellular $\mathrm{Ca}^{2+}$ release and SOCE in glutamatergic and aminergic neurons. Flight was rescued significantly by $d \mathrm{Orai}^{+}$expression in aminergic neurons $(D d c$;
Fig. $1 H)$ but not by expression in glutamatergic neurons (OK371; Fig. 1H). Moreover, significant rescue of flight was observed by $\mathrm{dOrai}^{+}$expression specifically in dopaminergic neurons $(\mathrm{TH}$; Fig. $1 \mathrm{H})$. Tyrosine hydroxylase $(\mathrm{TH})$ is a ratelimiting enzyme in dopamine synthesis and THGAL4 is expressed in a subset of $D d c G A L 4$-expressing neurons (FriggiGrelin et al., 2003). From the 30 flies of THGAL4-driven $\mathrm{dOrai}^{+}$tested, all but four could initiate flight. Their mean flight time was $7.1 \pm 1.2 \mathrm{~s}($ Fig. $1 H)$. These data suggest that GAL4-driven expression of $\mathrm{dOrai}^{+}$in dopaminergic neurons contributes to the rescue of flight in orai $^{3}$ mutants. Flight deficits in $\mathrm{orai}^{3}$ mutants and their rescue were further corroborated by electrophysiological recordings from the DLMs. Normal pattern of action potentials in response to an air puff were absent in orai $^{3}$ flies (Fig. $1 I, J$ ). Both $n S y b G A L 4$-driven and $D d c G A L 4$-driven overexpression of $d \mathrm{Orai}^{+}$rescued the reduced duration of DLM firing in $\operatorname{orai}^{3}$ homozygous flies to a significant extent (Fig. 1I,J). With DdcGAL4, characteristic tonic firing from the DLMs was observed in 1 of 15 randomly selected flies for $20-25$ s, 5 of 15 for $10-15$ s, and 9 of 15 for 3-5 s (Fig. 1I). Flies with THGAL4-driven $\mathrm{dOrai}^{+}$overexpression in dopaminergic neurons all initiated similar firing patterns, though these were not sustained beyond $10 \mathrm{~s}$ in a majority of flies (Fig. $1 \mathrm{I}, \mathrm{J}$ ). Thus overexpression of $\mathrm{dOrai}^{+}$in dopaminergic neurons of orai $^{3}$ mutants is sufficient for initiation of Drosophila flight.

\section{SOCE in dopaminergic neurons is abrogated by expression of} E180A, a dominant-negative form of dOrai

dOrai function in dopaminergic neurons was further investigated by cell-specific and stage-specific reduction of SOCE. For this purpose, we generated a Drosophila strain with an inducible dominant-negative form of $d O r a i$ in which the glutamate at residue 180 has been substituted with an alanine (Fig. 2A; Yeromin et al., 2006). Presence of dOrai ${ }^{\text {E180A }}$ (or E180A) subunits in dOrai tetramers abrogates channel function (Yeromin et al., 2006). We measured SOCE in TH-expressing neurons from $T H>E 180 \mathrm{~A}$ larvae and pupae by marking TH neurons with H2BmRFP (Langevin et al., 2005). SOCE was significantly reduced in both larval 
A

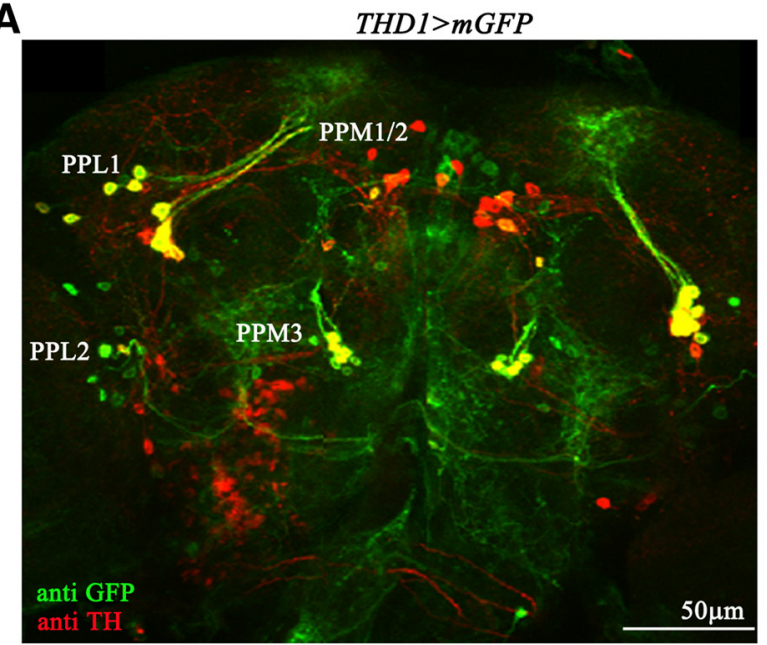

C

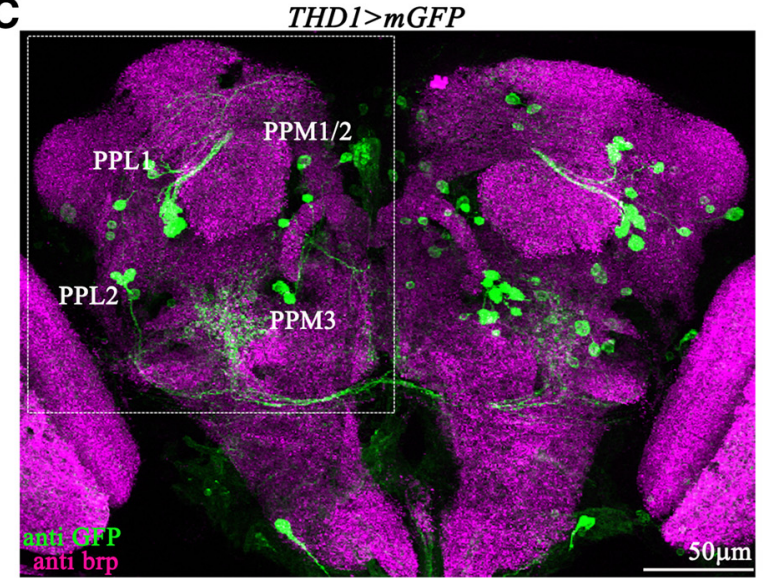

E

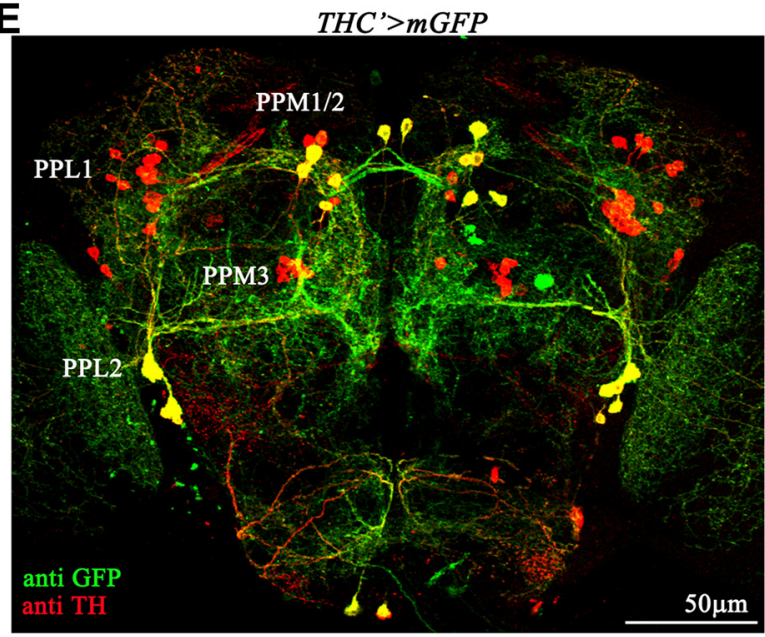

B

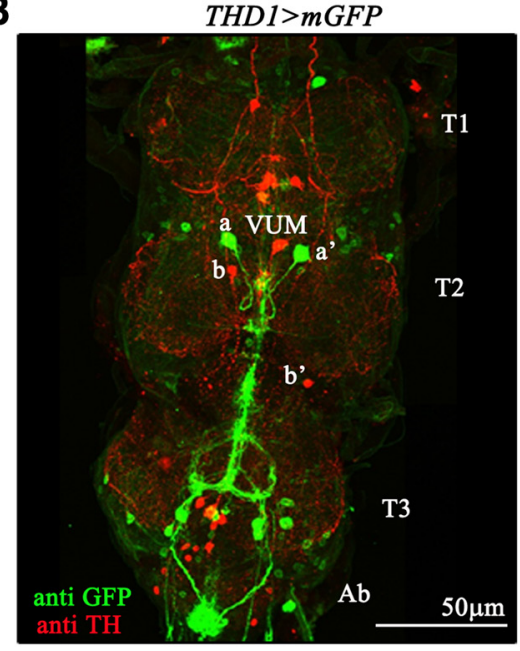

D

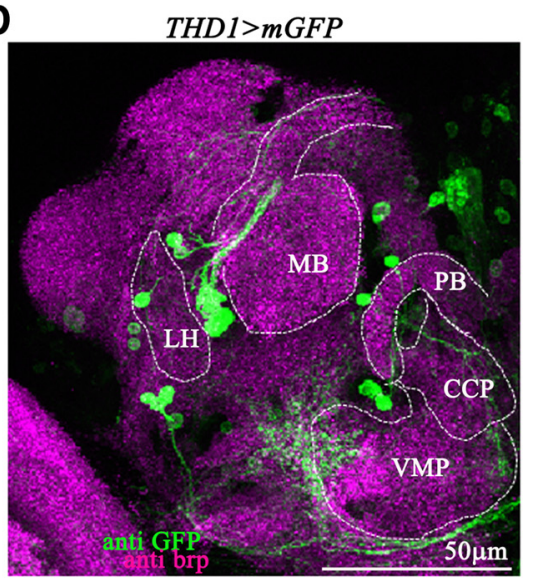

$F$

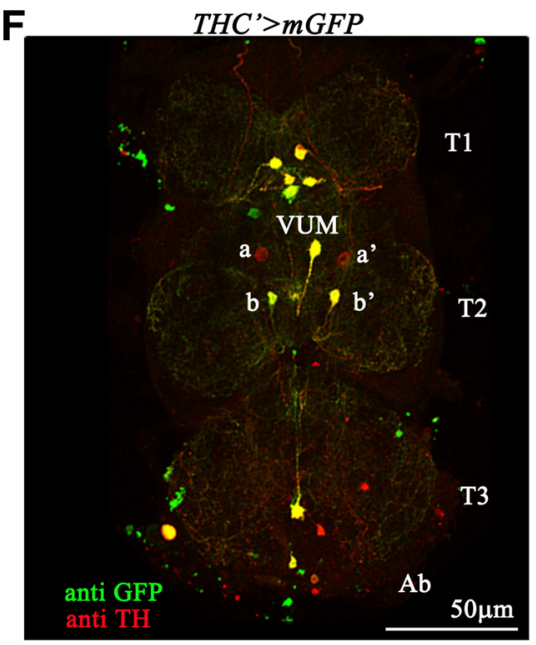

Figure 4. THD1 and THC'GAL4s express in different subsets of dopaminergic neurons. A, THD1GAL4-driven expression of mGFP in the posterior central brain region. Prominent dopaminergic neuron clusters of this region are marked. Dopaminergic neurons that express mGFP (PPL1 and PPM3) appear yellow. B, Expression pattern of THD1GAL4 in the ventral ganglia of the adult CNS. THD1GAL4 marks the a- $a^{\prime}$ neurons but does not mark ventral unpaired medial (VUM) and b- $b^{\prime}$ neurons in the T2 segment of the ventral ganglia. C, THD1GAL4-driven mGFP expression shown in the context of defined neuropil regions in the protocerebrum of an adult Drosophila brain. THD1 neurons are marked by anti-GFP and neuropil regions are marked by anti-brp. $\boldsymbol{D}$, Magnified image of part of the protocerebrum with cell bodies and their projections from the PPL1 cluster to the MB and from the PPM3 cluster [located near the ventromedial protocerebrum (VMP)] toward the central complex. LH, Lateral horn; CCP, caudal central protocerebrum; PB, protocerebral bridge. E, THC'GAL4-driven mGFP expression overlaps with anti-TH immunostaining of the PPL2 and PPM1/2 clusters (yellow) but not in the PPL1 and PPM3 clusters. F, THC'GAL4 marks the VUM and $b-b^{\prime}$ neurons but does not mark the $a-a^{\prime}$ neurons in the T2 segment.

and pupal dopaminergic neurons compared either with non- $\mathrm{TH}$ neurons (non-RFP cells from TH>E180A; $m R F P$ ) or with dopaminergic neurons obtained from control animals expressing only mRFP (TH>mRFP; Fig. $2 B-F)$. Expression of E180A did not affect store calcium release in dopaminergic neurons (Fig. 2B-F). Interestingly, the peak amplitude of SOCE in pupal neurons was significantly lower compared with larval neurons, suggesting stage-specific control of SOCE during pupal stages of neural cir- 

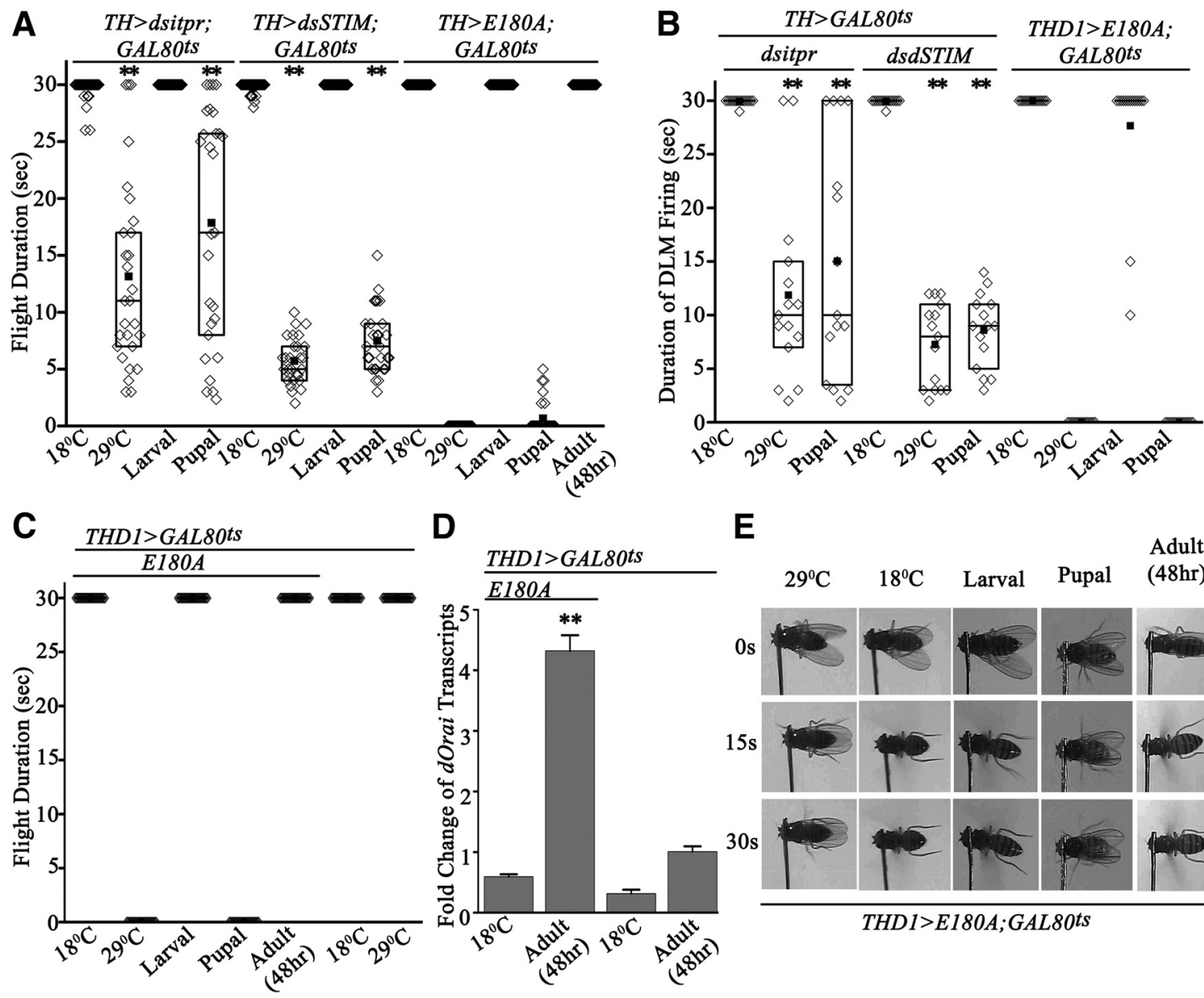

E

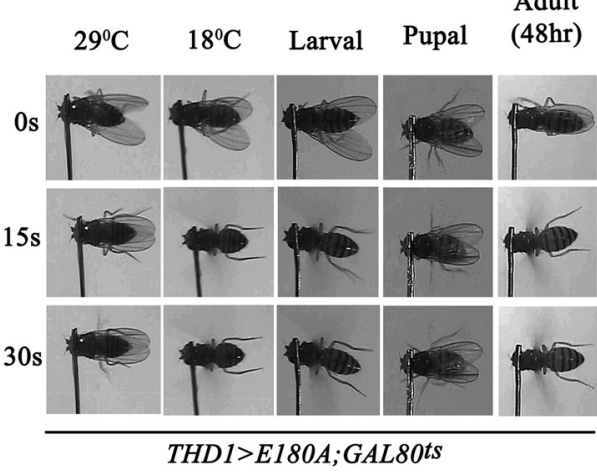

Figure 5. Reduction of SOCE in pupal dopaminergic neurons abolishes flight. $A$, Flight time of adult flies was measured after expression of either the appropriate RNAi strain (with UASDicer) or E180A by transfer to $29^{\circ} \mathrm{C}$ at the indicated stages of development (larval, pupal, and adult; $N=30$ ). For adult expression, the flies were transferred to $29^{\circ} \mathrm{C}$ immediately after eclosion. As controls, the strains were also grown at either 29 or $18^{\circ} \mathrm{C}$ throughout development. Flight times were compared with corresponding genetic controls grown at $18^{\circ} \mathrm{C}\left(\mathrm{N}=30\right.$; ${ }^{* *} \mathrm{p}<0.001$, Mann-Whitney test). $\boldsymbol{B}$, Duration of DLM firing trains. DLM recordings from flies of the indicated genotypes were obtained during an air puff-induced flight bout $\left(N=15\right.$; ${ }^{* *} p<0.001$, Mann-Whitney test). $\boldsymbol{C}$, Flight times were measured in THD1>E180A;GAL80 ts adult organisms by expressing E180A at either larval, pupal, or adult stages. D, Quantitative measurement (qPCR) of dOrai transcripts from dissected adult brains. Flies of the indicated genotypes were either maintained at $18^{\circ} \mathrm{C}$ throughout development and as adults or transferred to $29^{\circ} \mathrm{C}$ as adults immediately after eclosion for $48 \mathrm{~h}$. Expression of dOrai transcripts was significantly higher $\left(P_{\text {ANOVA }}<0.001\right)$ in brains from $T H D 1>E 180 A, G A L 80^{\text {ts }}$ flies after $48 \mathrm{~h}$ at $29^{\circ} \mathrm{C}$ compared with controls and flies grown at $18^{\circ} \mathrm{C}$. E, Snapshots obtained at the indicated time intervals from flight videos of air puff-stimulated flight bouts.

cuit maturation. Pupal neurons in Drosophila also exhibit spontaneous $\mathrm{Ca}^{2+}$ transients (Jiang et al., 2005). These data confirm that $E 180 A$ expression results in a cell-autonomous dominantnegative effect on SOCE in Drosophila neurons. The consequence of E180A expression in dopaminergic neurons on Drosophila flight was investigated next.

Dopaminergic neurons of the Drosophila flight circuit require calcium release through the $\mathrm{IP}_{3} \mathrm{R}$ and SOCE

SOCE is normally initiated in response to a physiological signal that first stimulates intracellular $\mathrm{Ca}^{2+}$ release from ER stores (Hogan et al., 2010; Smyth et al., 2010). To test whether Drosophila flight requires intracellular calcium release followed by SOCE in dopaminergic neurons, inducible RNAi strains for the $I P_{3} R$ (dsitpr), the ER $\mathrm{Ca}^{2+}$ sensor $\operatorname{dSTIM}$ (dsdSTIM), and the dominant-negative form of dOrai E180A were specifically expressed in dopaminergic neurons (Fig. 3). The dsitpr and dsdSTIM strains have been validated in previous publications (Agrawal et al., 2009; Venkiteswaran and Hasan, 2009). A partial flight deficit (19 $\pm 2.2 \mathrm{~s}$ mean flight time) was observed upon knockdown of the $\mathrm{IP}_{3} \mathrm{R}$ in dopaminergic neurons ( $T H>d$ sitpr), whereas a stronger flight deficit was observed upon dSTIM knockdown $(7.7 \pm 0.8 \mathrm{~s} ; T H>d s d S T I M)$, when compared with control flies $(28.2 \pm 0.6 \mathrm{~s}$; $T H>$ dicer; Fig. $3 A, B)$. The flight deficits observed corresponded to the variable durations of action potentials recorded from the DLMs during air puff-induced flight bouts. Duration of DLM firing during the short flight bouts was no different from the spike frequency of 6-10 Hz observed in controls (Fig. 3C). Variable flight durations observed among the knockdown flies are very likely due to differential efficacy of the RNAi strains in individual flies (Agrawal et al., 2009, 2010; Venkiteswaran and Hasan, 2009).

Whereas knockdown of intracellular $\mathrm{Ca}^{2+}$ signaling genes by RNAi resulted in partial flight deficits, expression of E180A in either aminergic neurons $(D d c>E 180 A)$ or dopaminergic neurons $(T H>E 180 A)$ resulted in complete loss of flight and flight-induced action potentials from the DLMs (Fig. 3A-C). Expression of E180A in serotonergic neurons (TRH $>E 180 A)$, which are also marked by the aminergic GAL4 ( $D d c$; Huser et al., 2012; Sadaf et al., 2012; Cassar et al., 2015), did not affect flight. Moreover, general motor function was not impaired by expression of E180A in dopaminergic neurons, as evident by their ability to climb normally (Fig. 3D). Together, these data support the idea that Orai function is specifically required in dopaminergic neurons of the flight circuit. Because 
A
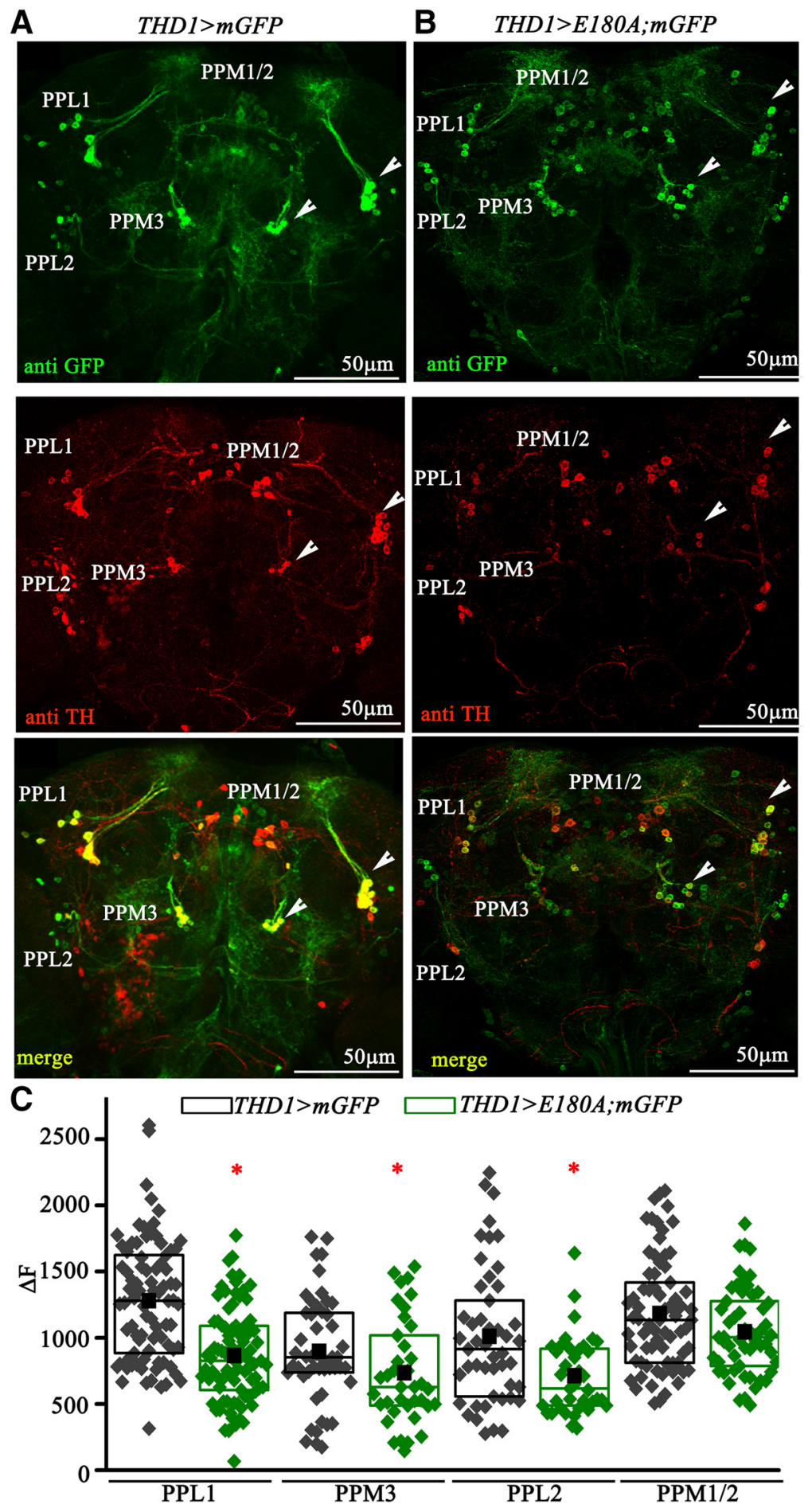

Figure 6. Loss of SOCE does not affect the pattern and number of dopaminergic neurons. $A$, Dopaminergic neuron clusters marked by anti-TH and anti-GFP immunostaining in the protocerebrum region of an adult Drosophila brain. Arrowheads indicate the PPL1 and PPM3 clusters of dopaminergic neurons. $\boldsymbol{B}$, Dopaminergic neuron clusters in the protocerebrum of $T H D 1>E 180 A$; $m$ GFP adults exhibit weak anti-TH immunostaining. GFP expression in cell bodies and their neurites appears similar to the control. C, Quantification of TH immunostaining in PPL1 ( $n \geq 83$ cells), PPL2 ( $n \geq 38$ cells), PPM2 ( $n \geq 55$ ) and PPM3 ( $n \geq 35$ ) neurons from THD1 $>$ GGFP (black) and THD1 $>$ E180A; $m$ GFP (green) adult Drosophila brains ( $N \geq 6$ brains). The $\Delta F$ values indicate $F-F_{\text {Basal, }}$ where the basal fluorescence $\left(F_{\text {Basal }}\right)$ of individual brain samples was subtracted from the total fluorescence $(F)$ of the individual neurons. One-way ANOVA was performed for each cell cluster followed by post hoc Tukey's test ( $\left.{ }^{*} P_{\text {ANOVA }}<0.05\right)$.

subset of dopaminergic neurons was confirmed by loss of flight in flies with E180A expression in dopaminergic neurons marked by THD1GAL4, THD'GAL4, and THD4GAL4 (Fig. 3 E,F). Partial flight deficits were also obtained by RNAi-mediated knockdown of the $I P_{3} R, d S T I M$, and dOrai in THD1-marked neurons (Fig. $3 E, F$ ). Interestingly, flies with E180A expression in the THC' dopaminergic neuron subdomain flew normally ( $T H C^{\prime}>E 180 A$; Fig. 3E,F). Therefore, we compared GFP-positive cells in the CNSs of flies with THGAL4-driven, THD1GAL4-driven, THD'GAL4-driven, THD4GAL4-driven, or THC'GAL4-driven UASmGFP expression (Table 1). This analysis suggests a requirement for SOCE in dopaminergic neurons of the protocerebrum posterior medial 3 (PPM3) and protocerebrum posterior lateral 1 (PPL1) clusters and a pair of neurons (a-a') in the T2 segment of the ventral ganglion, all of which are marked by THD1GAL4 but not by THC'GAL4 (Fig. 4). These data do not distinguish between the requirement for either one or all of these neuronal subsets for flight. Analysis of THD1 $>m G F P$ brains suggested that the PPM3 cluster projects to the central complex in the central brain, whereas the PPL1 cluster projects to the mushroom body $(\mathrm{MB})$ and the lateral horn (Fig. 4C,D). Projections of the PPM3 and PPL1 cluster to these regions of the central brain have been described earlier (Mao and Davis, 2009). Previous work has also confirmed that the THC'GAL4 does not mark a-a' cells of the ventral ganglion (Sadaf et al., 2015). Instead it marks three cells in the T2 segment, referred to as the b-b' pair and a single ventral unpaired medial neuron (Fig. $4 F$ ).

\section{Maturation of the flight circuit during pupal development requires SOCE}

Next, we asked whether loss of SOCE in dopaminergic neurons affects flightcircuit development and, if so, at what stage of development is SOCE required. To delineate the developmental stage for $\mathrm{IP}_{3} \mathrm{R}$, dSTIM, and dOrai requirement in dopaminergic neurons, we used the temporal and regional gene expression targeting system, in which a temperature shift to $29^{\circ} \mathrm{C}$ inactivates a mutant GAL80 ${ }^{\text {ts }}$ repressor protein, thereby activating GAL4 function (McGuire et al., 2004). Stagespecific knockdown of the $I P_{3} R$ and $d S$ $T I M$, as well as expression of E180A in dopaminergic neurons, followed by flight

TH is also expressed in the cuticle, where it is required for melanization (Wright, 1987), we tested flight after expression of E180A in multiple subdopaminergic neuronal domains that do not express in the cuticle (Liu et al., 2012). An essential requirement for SOCE in a tests in adults, revealed a requirement for intracellular calcium release and SOCE during pupal development (Fig. 5A-D). Pupal expression of E180A in dopaminergic neurons (TH>E180A; $G A L 80^{t s}$ and $\left.T H D 1>E 180 A ; G A L 80^{t s}\right)$ resulted in complete loss of 
flight (Fig. 4A-D). Pupal knockdown of either the $I P_{3} R$ or $d S T I M$ in all dopaminergic neurons (THGAL4) led to partial flight deficits with mean flight times of $17.8 \pm 1.9 \mathrm{~s}$ and $7.5 \pm 0.5 \mathrm{~s}$ respectively (Fig. 5A). Normal flight bouts were observed upon restricting the RNAi knockdowns and $E 180 A$ overexpression to larval stages, supporting SOCE function during pupal maturation of the flight circuit (Fig. $5 A-C, E)$. As controls, we tested RNAi and E180A-containing flies with the GAL80 ${ }^{t s}$ transgenes, grown at either $18^{\circ} \mathrm{C}$ (inactive GAL4) or $29^{\circ} \mathrm{C}$ (active GAL4) throughout development. Flight was not affected at $18^{\circ} \mathrm{C}$, and was significantly reduced (knockdowns) or absent $(E 180 A)$ at $29^{\circ} \mathrm{C}$ (Fig. $5 A-C, E$ ). Expression of E180A was also induced in adults for $48 \mathrm{~h}$ after eclosion. Normal flight durations were observed in these flies (Fig. $5 A, C, E$ ), indicating that $E 180 A$ expression does not affect dopaminergic neuron function during acute flight. Expression of the E180A transgene in dopaminergic neurons was confirmed in adults after $48 \mathrm{~h}$ of induction by measuring dOrai transcripts (Fig. 5D). Electrophysiological recordings from the DLMs of $I P_{3} R$ and dSTIM knockdowns and E180A-expressing flies, obtained after air puff stimulation of flight bouts, corroborated results from single-flight assays (Fig. 5B). From these data, we conclude that dopaminergic neurons of the Drosophila flight circuit require intracellular $\mathrm{Ca}^{2+}$ release through the $\mathrm{IP}_{3} \mathrm{R}$-mediated and $\mathrm{dSTIM} / \mathrm{dOrai}-$ mediated SOCE during pupal development, which enables their normal function in adult flight. These data do not exclude possible effects of E180A expression over longer periods on adult dopaminergic neurons. These are currently under investigation. Moreover, a requirement for $\mathrm{IP}_{3}$-mediated $\mathrm{Ca}^{2+}$ release and SOCE in dopaminergic neurons in adults is suggested by a significant difference between the flight times of $T H>d s i t p r$ flies with pupal knockdown compared with constant knockdown at $29^{\circ} \mathrm{C}$ (Fig. $5 A, B ; p<$ 0.01, Mann-Whitney test).

\section{SOCE controls the scale of TH}

upregulation in pupal dopaminergic neurons

To investigate possible underlying causes for loss of flight by reduced intracellular calcium signaling in dopaminergic neurons, the status of dopaminergic neurons in THD1>E180A fly brains was examined. Immunostaining with anti-TH revealed reduced immunoreactivity in the CNS of THD1 $>$ E180A adults compared
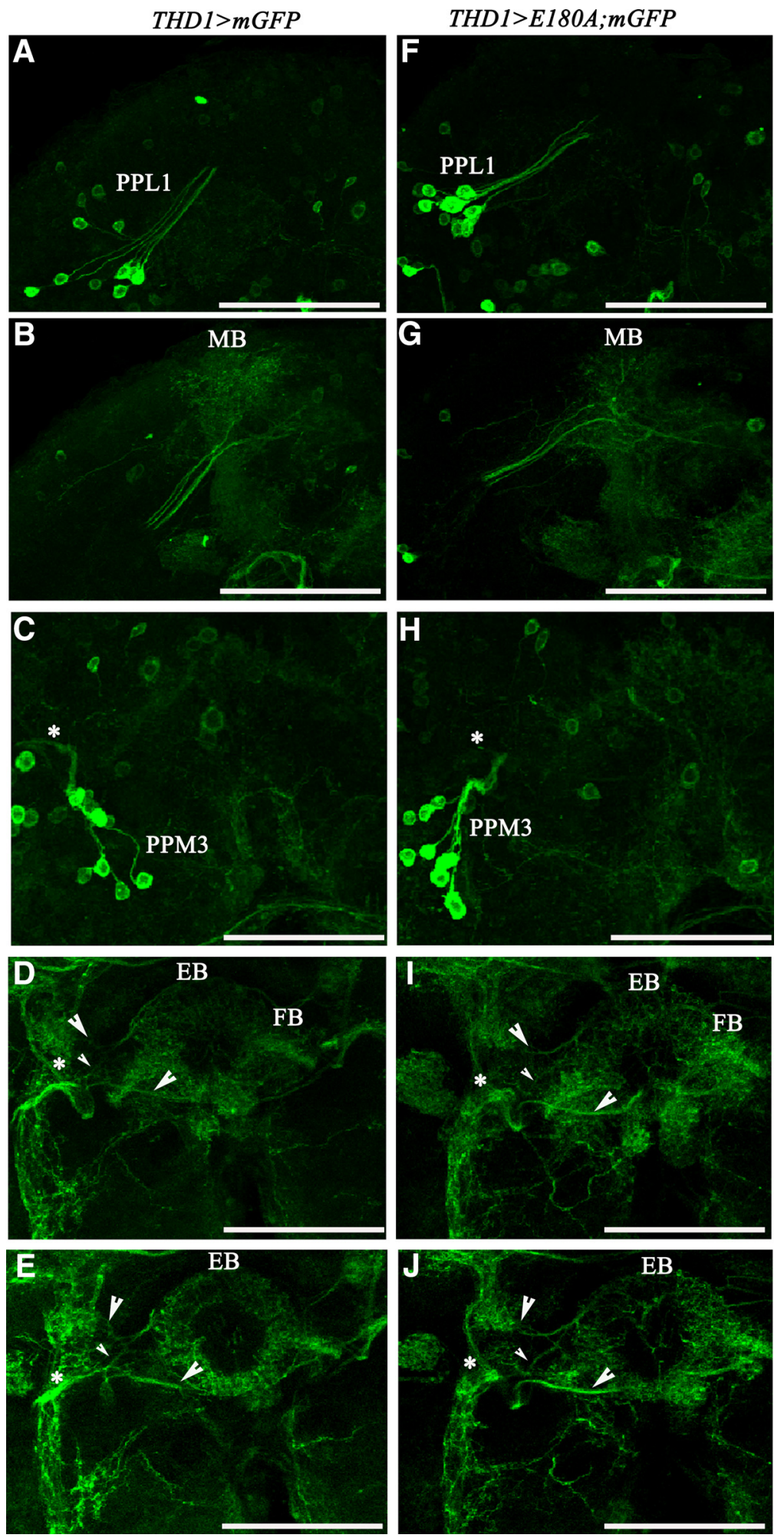

Figure 7. Comparison of projections from the dopaminergic PPL1 and PPM3 clusters in wild-type and SOCE-deficient brains. $A$, The PPL1 neuronal cluster and their projections in a THD1>mGFP adult brain (confocal stacks 74-98). $\boldsymbol{B}$, Projection from the PPL1 neurons enter the MB neuropil. The projections run from the posterior to the anterior (confocal section stacks 12-88). C, PPM3 cluster of dopaminergic neurons and their projections in a THD1> mGFP adult brain (confocal stacks 63-100). D, Projections from the PPM3 cluster (arrowheads) go toward the fan-shaped body (FB) and the EB (confocal stacks 41-52). $E$, Three PPM3 projections (arrowheads) enter the EB neuropil (confocal stacks 44-49). $\boldsymbol{F}$, PPL1 cluster and its projection in a THD1 $>$ E180A,mGFP brain. The image is a Z-stack of confocal sections 69-93 running from posterior to anterior. G, Projections of PPL1 neurons enter the MB neuropil (stacks 15-80). $\boldsymbol{H}$, PPM3 neurons in a THD1 > E180A; $m$ GFP brain (stacks 52-100). I, Three projections (arrowheads) of the PPM3 neurons head toward the EB. As in $\boldsymbol{D}$, one projection goes through the FB (stacks 37-55). J, Three major projections of the PPM3 neurons enter the EB neuropil (stacks 36-48). Asterisks refer to contiguous positions in the series of confocal stacks of $0.3 \mu \mathrm{m}$ each. Scale bars, $50 \mu \mathrm{m} . N=5$ brains for each genotype. 
A

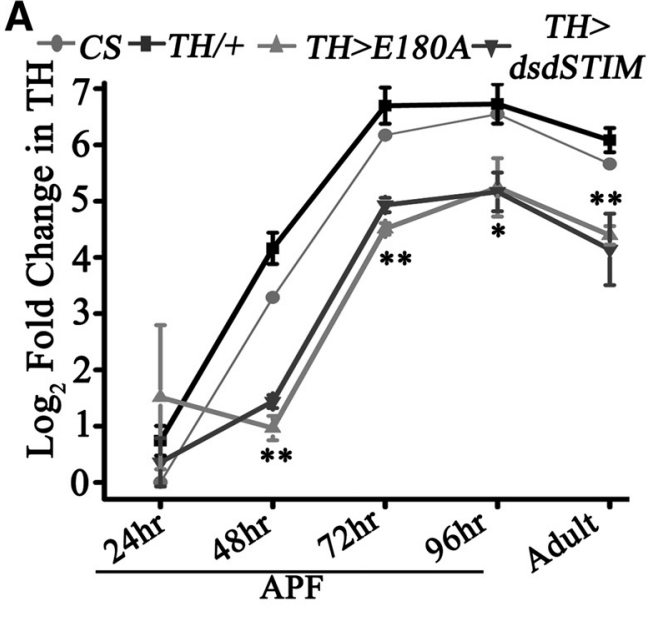

B

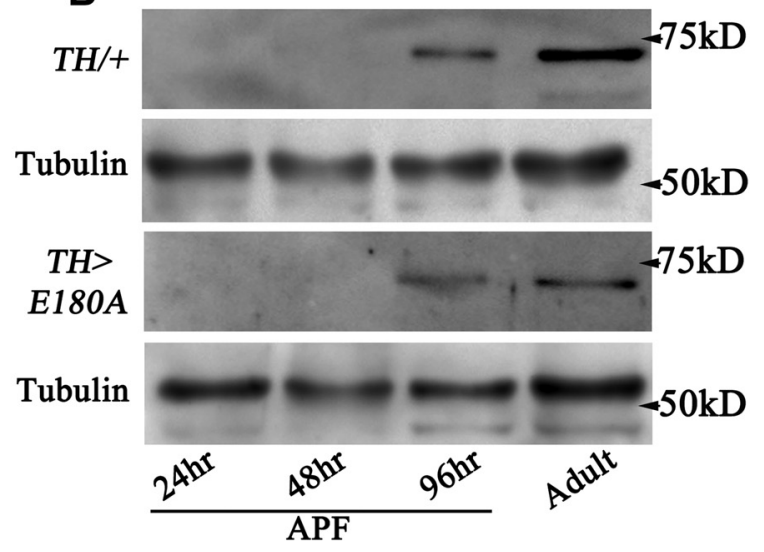

C

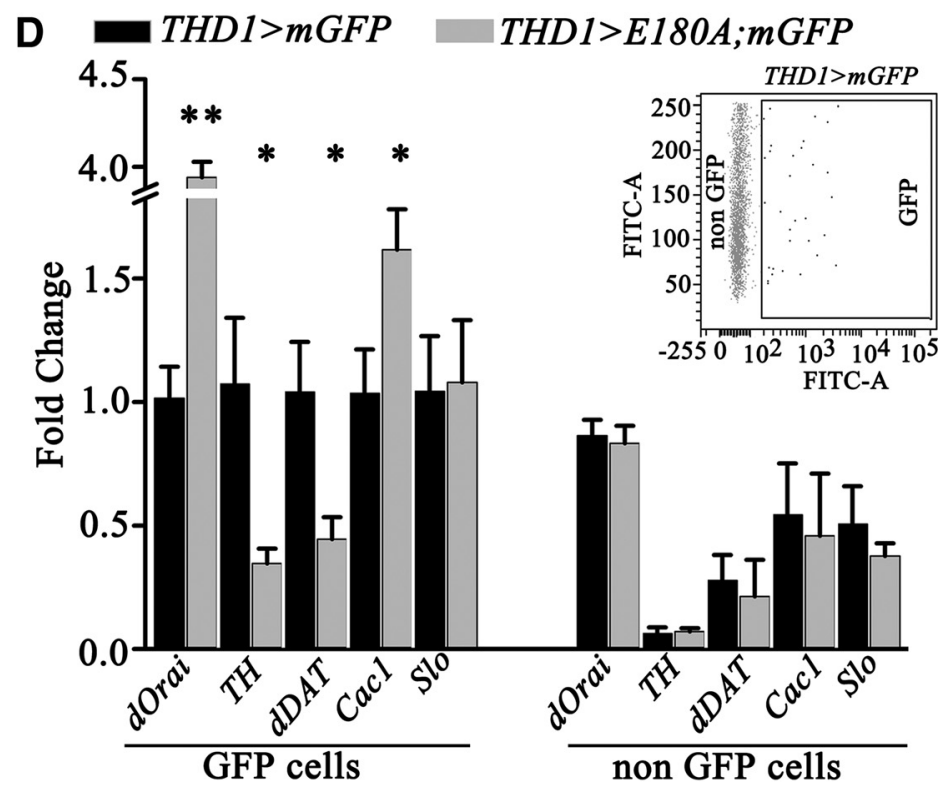

Figure 8. SOCE in pupae is required for normal levels of TH mRNA and protein in adult neurons. $A, T H$ transcript levels in pupal CNS of the indicated genotypes dissected at the indicated time points after puparium formation (APF). TH transcripts were normalized to $r p 49$ and are expressed as a $\log _{2 \text {-fold }}$ change from the wild-type strain (CS, gray) at $24 \mathrm{~h}$ APF ( $N \geq 3$, one-way ANOVA was performed for each time point followed by a post hoc Tukey's test; ${ }^{* *} p<0.05$ and $\left.{ }^{*} p<0.1\right)$. $\boldsymbol{B}$, TH protein levels are upregulated in parallel with $\mathrm{TH}$ transcripts in the CNS of developing pupae. TH protein levels were detected in Western blots with CNS lysates from control (TH/+) and experimental animals (TH $>$ E180A). The experiment was repeated twice. C, A representative Western blot of dissected adult CNSs from animals with E180A expression in THD1-marked neurons. E180A expression was allowed either throughout development ( $29^{\circ} \mathrm{C}$ ) or only during pupal stages (pupal) or prevented throughout development $\left(18^{\circ} \mathrm{C}\right)$. Quantification of three such blots where TH levels were normalized to that of tubulin are shown below $\left(N=3,{ }^{*} P_{t}\right.$ test $\left.<0.05\right)$. $\boldsymbol{D}, \mathbf{Q}$ Quantitative measurement ( $q P C R)$ of the indicated transcripts from GFP-positive and GFP-negative (non-GFP) cells. Significant differences were observed in GFP-positive cells obtained from the CNSs of TH>E180A; $m$ GFP (gray) animals compared with the control genotype, TH $>m G F P$ (black). Transcript levels appear unchanged between non-GFP cells from TH $>$ E180A; $m$ GFP (light gray) and control TH $>$ MGFP (dark gray) genotypes. Log 2 -fold changes were obtained as described in the methods. ( $\left.N=3,{ }^{*} P_{\text {ANOVA }}<0.05,{ }^{* *} P_{\text {ANOVA }}<0.01\right)$. The inset contains a representative dot plot of FACS analysis of single-cell suspensions from the dissected CNSs (48 h APF) of animals expressing GFP under the THD1 promoter. Approximately $1 \%$ of the cells were GFP positive. Both GFP-positive and GFP-negative cells were collected for transcript analysis.

with controls (Fig. 6A,B). Reduced immunoreactivity in individual neurons from THD1-marked clusters was quantified from multiple samples (Fig. 6C). A significant reduction was observed in cells of the PPL1, PPM3, and PPL2 clusters, where THD1 marks either all or a majority of neurons (Table 1; Fig. $4 A$ ). The PPL2 cluster is also marked by THC' (Fig. 4E). Hence, it is unlikely to contribute to the flight deficits observed with THD1driven expression of E180A. In contrast, only a few PPM1/2cluster neurons are marked by THD1 (Fig. 4A) and here the TH immunoreactivity was not reduced significantly (Fig. 6C). Despite reduced $\mathrm{TH}$ immunoreactivity, the number of $\mathrm{TH}$-positive cells in the CNS of THD1>E180A; $m$ GFP animals was no different from that of THD1>mGFP controls. Specifically, the numbers of TH-positive neurons in CNSs of THD1>E180A; $m$ GFP animals in the PPM3 cluster (6-7), in the PPL1 cluster (10-11), and in the T2 segment of the ventral ganglion (a-a') were unchanged and were similar to the number in THD1>mGFP animals (Table 1). Next we investigated more carefully the status of neurite projections from the PPM3 clusters, the PPL1 clusters (Fig. 7), and the a-a' neurons (data not shown) in controls (Fig. $7 A-E$ ) and THD1 $>E 180 A$ animals (Fig. 7F-J). PPL1 cluster neurons project to the $\mathrm{MB}$ (Kong et al., 2010). These appeared no different between control and THD1>E180A flies (Fig. 7, compare $A, B, F, G)$. In the 6-7 brains analyzed from controls and THD1>E180A flies, neurite extensions from the PPL1 to the MB varied between two and four in each brain hemisphere. On quantification, the average number of projections obtained from $\geq 12$ brain hemispheres was $3 \pm 0.96$ for controls and $3 \pm 1$ for 
A
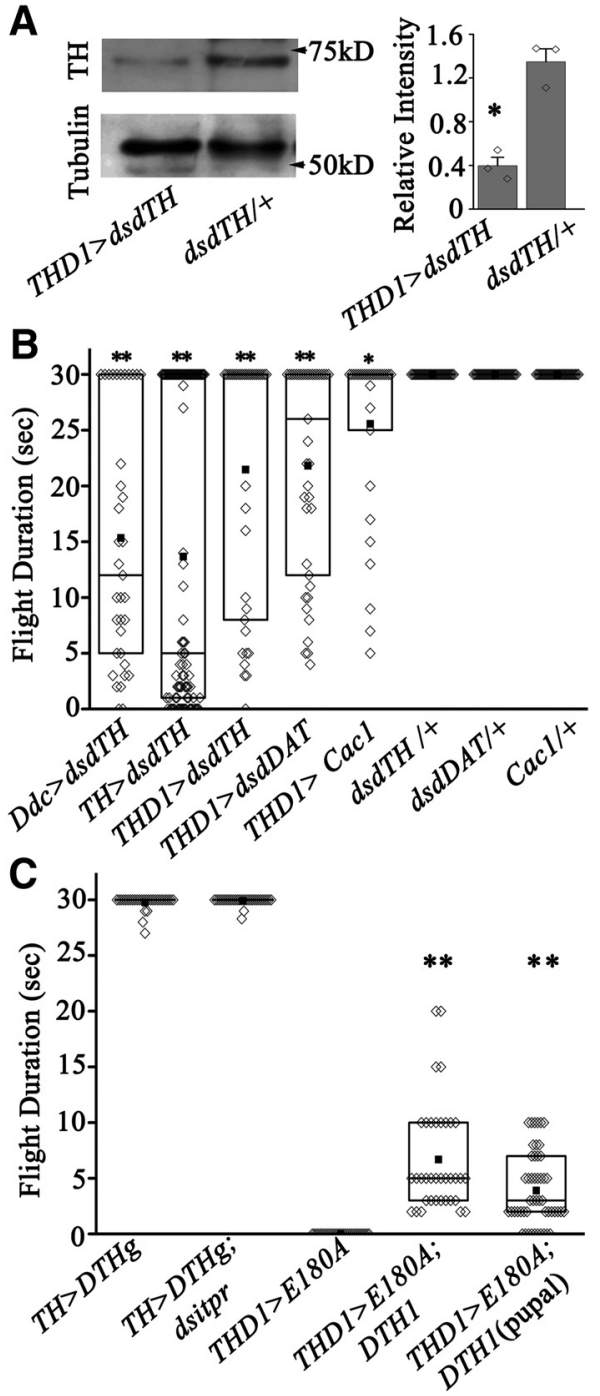

Figure 9. TH and dDAT are required for Drosophila flight. $A$, Western blot of TH levels in the dissected CNSs of flies with TH RNAi expression (THD1>dsDTH) and controls (dsDTH/+). Quantification of the mean relative intensity ( \pm SEM) of TH bands from the indicated genotypes with respect to tubulin is shown on the right $\left(N=3,{ }^{*} P_{t \text { test }}<0.05\right)$. $\boldsymbol{B}$, Flight time of adults with overexpression of the indicated transgenes under control of either Ddc, TH, or THD1 GAL4s. Flight times were compared with their corresponding controls $\left(N=30,{ }^{*} p<0.01\right.$, ${ }^{* *} p<$ 0.001. Mann-Whitney test). Significant flight deficits were obtained by knockdown of either $T H$ (dsdTH) or DAT (dsdDAT) and overexpression of Cac1. C, Flight times of flies of the indicated genotypes ( $N=30$; ${ }^{* *} p<0.001$, Mann-Whitney test).

TH $>$ E180A flies. Projections from the PPM3 cluster are more complex and lead toward different regions of the ellipsoid body (EB) in the central complex (Kong et al., 2010; Fig. 7C-E,H-J). One neurite projection goes through the fan-shaped body to the central region of the EB (Fig. 7D,I), whereas a second and third go directly to the anterior and posterior parts of the EB. All three projections were also observed in $T H>E 180 \mathrm{~A}$ adults (Fig. 7, compare $C-E, H-J)$. Thus loss of SOCE by E180A expression does not alter projection patterns of the PPL1 and PPM3 neurons. However, these data do not rule out more subtle changes in synapse structure that might arise within the $\mathrm{MB}$ and/or central complex neuropil regions.

Based on the reduced TH immunoreactivity observed and the absence of any visible anatomical change, we hypothesized that intracellular calcium signaling might affect $\mathrm{TH}$ mRNA and $\mathrm{TH}$ protein levels during pupal development. Indeed, levels of $T H$ transcripts increase dramatically in the CNS of 24-72 h pupae from control and Canton $S$ organisms (nearly a 70 -fold increase; Fig. $8 A$ ). This increase is dampened significantly ( $\sim 20$ fold) in CNS dissected from $T H>E 180 A$ and $T H>d s d S T I M$ pupae (Fig. $8 A$ ). Quantification of TH protein from brain lysates revealed similar results where intensity of $\mathrm{TH}$-specific bands at $96 \mathrm{~h}$ after puparium formation (APF) and in adults was significantly reduced in brains of TH>E180A organisms compared with controls (TH/+; Fig. $8 B$ ). TH levels were also reduced in the CNS of adult flies when expression of $E 180 A$ was restricted to pupal stages (THD1>E180A;GAL80 ${ }^{\text {ts }}$; Fig. $8 C$ ). Thus, SOCE in pupal dopaminergic neurons is required for upregulation of $T H$ mRNA and protein, and reduction in this upregulation correlates with loss of flight.

To test whether SOCE affects transcription of genes other than TH in dopaminergic neurons, we obtained GFP-marked neurons as well as non-GFP-marked neurons from brains of E180A; $m G F P$-expressing flies (THD1>E180A;mGFP) and controls $(T H D 1>m G F P)$ by FACS (Fig. $8 D$ ). RNA was isolated from sorted cells for measurement of transcript levels. In dopaminergic neurons with reduced SOCE, downregulation of $T H$ was accompanied by significant downregulation of dopamine transporter $(d D A T)$ transcript levels, whereas transcripts encoding a voltage-gated calcium channel Cac1 were upregulated (Fig. 8D). As a positive control, we measured $d O$ rai transcript levels, which exhibit significant upregulation upon E180A expression in GFPmarked neurons ( $T H D 1>E 180 A, m G F P)$, but remain constant in non-GFP-marked cells (Fig. 8D). Moreover, TH, dDAT, and Cacl transcripts remained constant in non-GFP-marked cells. Transcripts for a $\mathrm{Ca}^{2+}$-gated $\mathrm{K}^{+}$channel, Slowpoke (Slo), were unchanged by $E 180 \mathrm{~A}$ expression in dopaminergic neurons (Fig. 8D).

\section{Altered expression of TH, dDAT, and Cacl in dopaminergic neurons affects Drosophila flight}

To independently test the effect of altered TH levels on adult flight, we obtained an RNAi strain for Drosophila TH (dsDTH) and validated it by measuring $\mathrm{TH}$ protein in the CNS of THD1 $>d s D T H$ adults (Fig. 9A). We measured flight in flies with knockdown of TH in neurons marked by DdcGAL4, THGAL4, and THD1GAL4. All three knockdown strains exhibit significant flight deficits albeit to varying levels (Fig. 9B). Moreover, significant flight deficits were obtained upon knockdown of the dopamine transporter $(d D A T)$ and overexpression of $\mathrm{Cacl}$ in THD1-marked neurons (Fig. 9B). Next, we tested whether overexpression of $\mathrm{TH}$ could rescue flight deficits of SOCEcompromised flies. Overexpression of the complete genomic region of Drosophila TH (DTHg) (Friggi-Grelin et al., 2003) could rescue the flight deficit of $T H>d$ sitpr flies (compare Figs. 3A, 9C). Overexpression of a DTH cDNA (DTH1) encoding the neuronal isoform (Friggi-Grelin et al., 2003) either through development, or in pupae alone, could rescue flight initiation of THD1 $>$ E180A animals to a significant extent (compare Figs. 3E, 9C). The rescue by DTH1 argues against changes in synaptic structure (that might affect dopamine release) as a primary cause of the flight deficits observed; rather, it supports reduced TH transcription as a key feature of neurons with compromised SOCE, and normal $\mathrm{TH}$ levels as an essential requirement for flight initiation. However, this rescue was insufficient to maintain flight beyond 7-8 s (Fig. 9C). Thus TH overexpression is probably insufficient to restore expression of other SOCE-regulated genes. Upregulation of the voltage-gated $\mathrm{Ca}^{2+}$ channel subunit, encoded by Cacl (Fig. 8D), 
is one such factor that would alter membrane excitability and therefore neural function during adult flight.

\section{Discussion}

Null mutants in $\mathrm{Ca}^{2+}$-signaling genes, such as Orai, STIM, and $I P_{3} R$, have pleiotropic effects during development, which frequently result in either defective development or early lethality of the organism (Venkatesh and Hasan, 1997; Joshi et al., 2004). Consequently, identification of molecular and cellular mechanisms regulated by intracellular $\mathrm{Ca}^{2+}$ signaling and SOCE in differentiated neurons requires genetic tools that can be restricted in their spatiotemporal expression. We addressed this problem by first studying a hypomorphic allele of the single Drosophila Orai gene followed by developing a dominant-negative Orai transgene whose expression could be manipulated in a stage-specific and tissue-specific manner. Temporal expression of the dominant-negative E180A transgene established that SOCE requirement in dopaminergic neurons is during pupal maturation of the flight circuit. Moreover, loss of SOCE does not affect the number of dopaminergic neurons; rather, it alters their transcriptional profile by affecting key molecules, such as TH, DAT, and Cacl or Dmca1A/Ca 2 . Whereas downregulation of $T H$ and $D A T$ expression would reduce synaptic neurotransmitter release, upregulated Cac1 levels are likely to affect synaptic homeostasis (Lee et al., 2014). It is likely that SOCE regulates expression of other genes in addition to TH,DAT, and Cacl. Thus SOCE is required for attaining the final functional state of dopaminergic flight neurons in the adult brain.

Further analysis of dopaminergic subdomains that require SOCE support one or both of two central brain clusters (PPM3 and PPL1) and a pair of neurons in the second thoracic segment (T2) of the ventral ganglion ( $\mathrm{a}-\mathrm{a}$ ') as necessary for flight. Interestingly, recent work has demonstrated a role for the a-a' pair of neurons in modulation of bilateral wing-coordinating motor neurons during initiation and cessation of Drosophila flight (Sadaf et al., 2015). The PPM3 and PPL1 clusters have not been implicated in flight control earlier. Cell bodies of the PPM3 cluster lie in the vicinity of the ventromedial protocerebrum neuropil and project to the EB of the central complex. As demonstrated recently, these regions form part of the auditory/mechanosensory module in the fly brain and might thus constitute centers for control of voluntary flight initiation (Shih et al., 2015). Maintenance of flight bouts over several minutes also requires synaptic activity in another cluster of central brain dopaminergic neurons, the protocerebral anteriomedial (PAM) cluster (Agrawal and Hasan, 2015). Both the PPL1 and PAM clusters project to a higher brain center, the MB (Mao and Davis, 2009; Aso et al., 2012), which is required for reinforcement of aversive and appetitive responses to olfactory stimuli (Waddell, 2013). In the natural environment, the behavioral response to such olfactory stimuli is likely to be flight. Thus regions of the MB may function as a center for integration of flight behavior with other relevant sensory inputs, such as olfaction.

TH levels appear reduced in all dopaminergic neurons with compromised SOCE. Dopaminergic interneurons in Drosophila are known to modulate several behaviors, including climbing (Riemensperger et al., 2013), sleep bouts (Liu et al., 2012), olfactory learning (Waddell, 2013), water reward (Lin et al., 2014), aversive memory formation (Schwaerzel et al., 2003), and amino acid sensing in larvae (Bjordal et al., 2014). The absence of climbing deficits in $T H>E 180 \mathrm{~A}$ adults (Fig.
$3 D$ ) suggests that dopaminergic flight-circuit neurons may be more sensitive to changes in dopamine signaling compared with other neural circuits. Moreover, the absence of flight deficits by either knockdown of dSTIM (Sadaf et al., 2012) or expression of E180A in serotonergic neurons (Fig. 3A) supports SOCE as an integral signaling component of maturing dopaminergic flight interneurons. Sensitivity of dopaminergic flight interneurons to E180A expression may in part be due to reduced dOrai RNA/protein in these neurons and needs further investigation. Other behavioral deficits in $T H>E 180 \mathrm{~A}$ flies also need investigation.

In a previous study, flight deficits were obtained upon knockdown of dOrai in glutamatergic neurons of Drosophila (Venkiteswaran and Hasan, 2009). However, orai ${ }^{3}$ mutants are not rescued by $\mathrm{dOrai}^{+}$expression in glutamatergic neurons (Fig. $1 H$ ). It is likely that both dopaminergic and glutamatergic neurons of the flight circuit require Orai function, but rescue of SOCE in glutamatergic neurons may not be sufficient to restore flight if their function is downstream of dopaminergic neurons. Simultaneous rescue from dopaminergic and glutamatergic neurons has not been attempted but should help resolve this issue. Moreover, partial rescue of flight in orai $^{3}$ mutants by $\mathrm{dOrai}^{+}$expression in dopaminergic neurons supports the existence of additional SOCE-requiring neuronal subsets for flight-circuit development and/or function.

At this stage, we cannot state conclusively whether $48 \mathrm{~h}$ of E180A induction in adults is sufficient to alter TH levels in dopaminergic neurons. Thus it will also be of interest to understand the role of SOCE for adult flight-circuit function over longer periods, such as days or weeks. During pupal development, upregulation of Dmcala/Cacl in dopaminergic neurons with reduced SOCE (Fig. $8 D$ ) might serve as a compensatory mechanism for entry of extracellular calcium and consequent refilling of intracellular stores. Moreover, viability of SOCE-compromised neurons in pupae may depend on such a compensatory mechanism. In adults, however, Cac1 overexpression is likely to affect membrane excitability and synaptic strength, thus altering the response from dopaminergic neurons of the flight circuit (Lee et al., 2014). Collectively, our data show SOCE-dependent transcriptional regulation of dopaminergic neurons during development with subsequent consequences on the function of an adult neural circuit. Normal function of dopaminergic neurons is essential for motor coordination in vertebrates and their malfunction can lead to neurodegenerative disorders, such as Parkinson's disease (Chan et al., 2009). Work on treatments for such conditions might benefit from a better understanding of the signaling mechanism(s) through which SOCE regulates TH transcription in development and the function of differentiated adult dopaminergic neurons.

\section{References}

Agrawal T, Hasan G (2015) Maturation of a central brain flight circuit in Drosophila requires Fz2/Ca ${ }^{2+}$ signaling. Elife 4:e07046. CrossRef Medline

Agrawal N, Padmanabhan N, Hasan G (2009) Inositol 1,4,5-trisphosphate receptor function in Drosophila insulin producing cells. PLoS One 4:e6652. CrossRef Medline

Agrawal N, Venkiteswaran G, Sadaf S, Padmanabhan N, Banerjee S, Hasan G (2010) Inositol 1,4,5-trisphosphate receptor and dSTIM function in Drosophila insulin-producing neurons regulates systemic intracellular calcium homeostasis and flight. J Neurosci 30:1301-1313. CrossRef Medline

Agrawal T, Sadaf S, Hasan G (2013) A genetic RNAi screen for IP(3)/ $\mathrm{Ca}(2)(+)$ coupled GPCRs in Drosophila identifies the PdfR as a regulator of insect flight. PLoS Genet 9:e1003849. CrossRef Medline

Aso Y, Herb A, Ogueta M, Siwanowicz I, Templier T, Friedrich AB, Ito K, 
Scholz H, Tanimoto H (2012) Three dopamine pathways induce aversive odor memories with different stability. PLoS Genet 8:e1002768. CrossRef Medline

Banerjee S, Lee J, Venkatesh K, Wu CF, Hasan G (2004) Loss of flight and associated neuronal rhythmicity in inositol 1,4,5-trisphosphate receptor mutants of Drosophila. J Neurosci 24:7869-7878. CrossRef Medline

Berridge MJ (1998) Neuronal calcium signaling. Neuron 21:13-26. CrossRef Medline

Bjordal M, Arquier N, Kniazeff J, Pin JP, Léopold P (2014) Sensing of amino acids in a dopaminergic circuitry promotes rejection of an incomplete diet in Drosophila. Cell 156:510-521. CrossRef Medline

Borodinsky LN, Root CM, Cronin JA, Sann SB, Gu X, Spitzer NC (2004) Activity-dependent homeostatic specification of transmitter expression in embryonic neurons. Nature 429:523-530. CrossRef Medline

Brand AH, Perrimon N (1993) Targeted gene expression as a means of altering cell fates and generating dominant phenotypes. Development 118: 401-415. Medline

Cassar M, Issa AR, Riemensperger T, Petitgas C, Rival T, Coulom H, IchéTorres M, Han KA, Birman S (2015) A dopamine receptor contributes to paraquat-induced neurotoxicity in Drosophila. Hum Mol Genet 24: 197-212. CrossRef Medline

Chakraborty S, Hasan G (2012) Functional complementation of Drosophila itpr mutants by rat Itpr1. J Neurogenet 26:328-337. CrossRef Medline

Chan CS, Gertler TS, Surmeier DJ (2009) Calcium homeostasis, selective vulnerability and Parkinson's disease. Trends Neurosci 32:249-256. CrossRef Medline

Cuttell L, Vaughan A, Silva E, Escaron CJ, Lavine M, Van Goethem E, Eid JP, Quirin M, Franc NC (2008) Undertaker, a Drosophila junctophilin, links Draper-mediated phagocytosis and calcium homeostasis. Cell 135: 524-534. CrossRef Medline

Feske S, Gwack Y, Prakriya M, Srikanth S, Puppel SH, Tanasa B, Hogan PG, Lewis RS, Daly M, Rao A (2006) A mutation in Orail causes immune deficiency by abrogating CRAC channel function. Nature 441:179-185. CrossRef Medline

Friggi-Grelin F, Iché M, Birman S (2003) Tissue-specific developmental requirements of Drosophila tyrosine hydroxylase isoforms. Genesis 35: 260-269. CrossRef Medline

Hartmann J, Karl RM, Alexander RP, Adelsberger H, Brill MS, Rühlmann C, Ansel A, Sakimura K, Baba Y, Kurosaki T, Misgeld T, Konnerth A (2014) STIM1 controls neuronal $\mathrm{Ca}(2)(+)$ signaling, mGluR1-dependent synaptic transmission, and cerebellar motor behavior. Neuron 82:635-644. CrossRef Medline

Hogan PG, Lewis RS, Rao A (2010) Molecular basis of calcium signaling in lymphocytes: STIM and ORAI. Annu Rev Immunol 28:491-533. CrossRef Medline

Huser A, Rohwedder A, Apostolopoulou AA, Widmann A, Pfitzenmaier JE, Maiolo EM, Selcho M, Pauls D, von Essen A, Gupta T, Sprecher SG, Birman S, Riemensperger T, Stocker RF, Thum AS (2012) The serotonergic central nervous system of the Drosophila larva: anatomy and behavioral function. PLoS One 7:e47518. CrossRef Medline

Jiang SA, Campusano JM, Su H, O’Dowd DK (2005) Drosophila mushroom body Kenyon cells generate spontaneous calcium transients mediated by PLTX-sensitive calcium channels. J Neurophysiol 94:491-500. CrossRef Medline

Joshi R, Venkatesh K, Srinivas R, Nair S, Hasan G (2004) Genetic dissection of itpr gene function reveals a vital requirement in aminergic cells of Drosophila larvae. Genetics 166:225-236. CrossRef Medline

Kong EC, Woo K, Li H, Lebestky T, Mayer N, Sniffen MR, Heberlein U, Bainton RJ, Hirsh J, Wolf FW (2010) A pair of dopamine neurons target the D1-like dopamine receptor DopR in the central complex to promote ethanol-stimulated locomotion in Drosophila. PLoS One 5:e9954. CrossRef Medline

Lalonde J, Saia G, Gill G (2014) Store-operated calcium entry promotes the degradation of the transcription factor Sp4 in resting neurons. Sci Signal 7:ra51. CrossRef Medline

Langevin J, Le Borgne R, Rosenfeld F, Gho M, Schweisguth F, Bellaïche Y (2005) Lethal giant larvae controls the localization of notch-signaling regulators numb, neuralized, and Sanpodo in Drosophila sensory-organ precursor cells. Curr Biol 15:955-962. CrossRef Medline

Lee J, Ueda A, Wu CF (2014) Distinct roles of Drosophila cacophony and DmcalD $\mathrm{Ca}(2+)$ channels in synaptic homeostasis: genetic interactions with slowpoke $\mathrm{Ca}(2+)$-activated $\mathrm{BK}$ channels in presynaptic excitability and postsynaptic response. Dev Neurobiol 74:1-15. CrossRef Medline
Lee T, Lee A, Luo L (1999) Development of the Drosophila mushroom bodies: sequential generation of three distinct types of neurons from a neuroblast. Development 126:4065-4076. Medline

Lin S, Owald D, Chandra V, Talbot C, Huetteroth W, Waddell S (2014) Neural correlates of water reward in thirsty Drosophila. Nat Neurosci 17:1536-1542. CrossRef Medline

Liu Q, Liu S, Kodama L, Driscoll MR, Wu MN (2012) Two dopaminergic neurons signal to the dorsal fan-shaped body to promote wakefulness in Drosophila. Curr Biol 22:2114-2123. CrossRef Medline

Lorentzos P, Kaiser T, Kennerson ML, Nicholson GA (2003) A rapid and definitive test for Charcot-Marie-Tooth 1A and hereditary neuropathy with liability to pressure palsies using multiplexed real-time PCR. Genet Test 7:135-138. CrossRef Medline

Lundell MJ, Hirsh J (1994) Temporal and spatial development of serotonin and dopamine neurons in the Drosophila CNS. Dev Biol 165:385-396. CrossRef Medline

Mao Z, Davis RL (2009) Eight different types of dopaminergic neurons innervate the Drosophila mushroom body neuropil: anatomical and physiological heterogeneity. Front Neural Circuits 3:5. Medline

McGuire SE, Mao Z, Davis RL (2004) Spatiotemporal gene expression targeting with the TARGET and gene-switch systems in Drosophila. Sci STKE 2004:pl6. Medline

Miller DL, Ballard SL, Ganetzky B (2012) Analysis of synaptic growth and function in Drosophila with an extended larval stage. J Neurosci 32: 13776-13786. CrossRef Medline

Pfeiffer BD, Jenett A, Hammonds AS, Ngo TT, Misra S, Murphy C, Scully A, Carlson JW, Wan KH, Laverty TR, Mungall C, Svirskas R, Kadonaga JT, Doe CQ, Eisen MB, Celniker SE, Rubin GM (2008) Tools for neuroanatomy and neurogenetics in Drosophila. Proc Natl Acad Sci U S A 105: 9715-9720. CrossRef Medline

Riemensperger T, Issa AR, Pech U, Coulom H, Nguyen MV, Cassar M, Jacquet M, Fiala A, Birman S (2013) A single dopamine pathway underlies progressive locomotor deficits in a Drosophila model of Parkinson disease. Cell Rep 5:952-960. CrossRef Medline

Ryglewski S, Kilo L, Duch C (2014) Sequential acquisition of cacophony calcium currents, sodium channels and voltage-dependent potassium currents affects spike shape and dendrite growth during postembryonic maturation of an identified Drosophila motoneuron. Eur J Neurosci 39: 1572-1585. CrossRef Medline

SadafS, Birman S, Hasan G (2012) Synaptic activity in serotonergic neurons is required for air-puff stimulated flight in Drosophila melanogaster. PLoS One 7:e46405. CrossRef Medline

Sadaf S, Reddy OV, Sane SP, Hasan G (2015) Neural control of wing coordination in flies. Curr Biol 25:80-86. CrossRef Medline

Schwaerzel M, Monastirioti M, Scholz H, Friggi-Grelin F, Birman S, Heisenberg M (2003) Dopamine and octopamine differentiate between aversive and appetitive olfactory memories in Drosophila. J Neurosci 23: 10495-10502. Medline

Shih CT, Sporns O, Yuan SL, Su TS, Lin YJ, Chuang CC, Wang TY, Lo CC, Greenspan RJ, Chiang AS (2015) Connectomics-based analysis of information flow in the Drosophila brain. Curr Biol 25:1249-1258. CrossRef Medline

Skibinska-Kijek A, Wisniewska MB, Gruszczynska-Biegala J, Methner A, Kuznicki J (2009) Immunolocalization of STIM1 in the mouse brain. Acta Neurobiol Exp (Wars) 69:413-428. Medline

Smyth JT, Hwang SY, Tomita T, DeHaven WI, Mercer JC, Putney JW (2010) Activation and regulation of store-operated calcium entry. J Cell Mol Med 14:2337-2349. CrossRef Medline

Somasundaram A, Shum AK, McBride HJ, Kessler JA, Feske S, Miller RJ, Prakriya M (2014) Store-operated CRAC channels regulate gene expression and proliferation in neural progenitor cells. J Neurosci 34:91079123. CrossRef Medline

Spitzer NC (2002) Activity-dependent neuronal differentiation prior to synapse formation: the functions of calcium transients. J Physiol Paris 96:73-80. CrossRef Medline

Timmerman C, Suppiah S, Gurudatta BV, Yang J, Banerjee C, Sandstrom DJ, Corces VG, Sanyal S (2013) The Drosophila transcription factor Adf-1 (nalyot) regulates dendrite growth by controlling FasII and Staufen expression downstream of CaMKII and neural activity. J Neurosci 33: 11916-11931. CrossRef Medline

Venkatesh K, Hasan G (1997) Disruption of the IP3 receptor gene of Dro- 
sophila affects larval metamorphosis and ecdysone release. Curr Biol 7:500-509. CrossRef Medline

Venkiteswaran G, Hasan G (2009) Intracellular Ca2+ signaling and storeoperated Ca2 + entry are required in Drosophila neurons for flight. Proc Natl Acad Sci U S A 106:10326-10331. CrossRef Medline

Vig M, Peinelt C, Beck A, Koomoa DL, Rabah D, Koblan-Huberson M, Kraft S, Turner H, Fleig A, Penner R, Kinet JP (2006) CRACM1 is a plasma membrane protein essential for store-operated $\mathrm{Ca} 2+$ entry. Science 312 : 1220-1223. CrossRef Medline

Vonhoff F, Kuehn C, Blumenstock S, Sanyal S, Duch C (2013) Temporal coherency between receptor expression, neural activity and AP-1dependent transcription regulates Drosophila motoneuron dendrite development. Development 140:606-616. CrossRef Medline

Waddell S (2013) Reinforcement signalling in Drosophila; dopamine does it all after all. Curr Opin Neurobiol 23:324-329. CrossRef Medline
Wright TR (1987) The genetics of biogenic amine metabolism, sclerotization, and melanization in Drosophila melanogaster. Adv Genet 24:127222. CrossRef Medline

Wu CF, Suzuki N, Poo MM (1983) Dissociated neurons from normal and mutant Drosophila larval central nervous system in cell culture. J Neurosci 3:1888-1899. Medline

Wu J, Shih HP, Vigont V, Hrdlicka L, Diggins L, Singh C, Mahoney M, Chesworth R, Shapiro G, Zimina O, Chen X, Wu Q, Glushankova L, Ahlijanian M, Koenig G, Mozhayeva GN, Kaznacheyeva E, Bezprozvanny I (2011) Neuronal store-operated calcium entry pathway as a novel therapeutic target for Huntington's disease treatment. Chem Biol 18:777793. CrossRef Medline

Yeromin AV, Zhang SL, Jiang W, Yu Y, Safrina O, Cahalan MD (2006) Molecular identification of the CRAC channel by altered ion selectivity in a mutant of Orai. Nature 443:226-229. CrossRef Medline 